\title{
Alberto ARABiA
}

\section{Cohomologie T-équivariante de la variété de drapeaux d'un groupe de Kač-Moody}

\author{
Bulletin de la S. M. F., tome 117, no 2 (1989), p. 129-165 \\ <http://www.numdam.org/item?id=BSMF_1989_117_2_129_0>
}

(C) Bulletin de la S. M. F., 1989, tous droits réservés.

L'accès aux archives de la revue «Bulletin de la S. M. F. » (http: //smf.emath.fr/Publications/Bulletin/Presentation.html) implique l'accord avec les conditions générales d'utilisation (http://www.numdam.org/ conditions). Toute utilisation commerciale ou impression systématique est constitutive d'une infraction pénale. Toute copie ou impression de ce fichier doit contenir la présente mention de copyright.

\section{Numdam}




\title{
COHOMOLOGIE T-ÉQUIVARIANTE DE LA VARIÉTÉ DE DRAPEAUX D'UN GROUPE DE KAČ-MOODY
}

\author{
PAR
}

\author{
Alberto ARABIA $\left({ }^{*}\right)$
}

RÉsumé. - On définit des opérateurs $\mathcal{A}_{i}$ de Bernstein-Gel'fand-Gel'fand sur la cohomologie $T$-équivariante entière $H_{T}^{*}(\mathcal{F})$ de la variété de drapeaux $\mathcal{F}=G / B$ d'un groupe de Kač-Moody $G$. En intégrant sur les variétés de Schubert de $\mathcal{F}$, on caractérise une famille $\left\{\mathcal{L}_{w}\right\}_{w \in W}$ de formes $H_{T}^{*}(\cdot)$-linéaires sur $H_{T}^{*}(\mathcal{F})$, base du dual de $H_{T}^{*}(\mathcal{F})$. Ces formes canoniques sont liées aux opérateurs $\mathcal{A}_{i}$ par l'égalité $\mathcal{L}_{w r_{i}}=\mathcal{L}_{w} \mathcal{A}_{i}$ lorsque $w r_{i}>w$, ce qui entraîne le caractère intrinsèque des composées $\mathcal{A}_{w}$ des opérateurs en question. On prouve que les $\mathcal{A}_{w}$ peuvent être obtenus par intégration sur les fibres de certaines fibrations au-dessus de $\mathcal{F}$.

Par restriction au sous-espace $W$ des points fixes de $T$ dans $\mathcal{F}$, on donne un homomorphisme injectif $\Theta$ de $H_{T}^{*}(\mathcal{F})$ dans l'algèbre $F(W ; Q)$ de toutes les applications définies sur $W$ à valeurs dans le corps $Q$ des fractions rationnelles de l'algèbre de polynômes $S=\mathbb{Z}\left[\alpha_{1}, \ldots, \alpha_{n}\right]$, où $\left\{\alpha_{1}, \ldots, \alpha_{n}\right\}$ dénote le système des racines simples de l'algèbre de Lie de $G$. Des formules explicites pour les localisations des formes $\mathcal{L}_{w}$ sur $F(W ; Q)$ sont données. On détermine de même les localisations $A_{i}$ des $\mathcal{A}_{i} \operatorname{sur} F(W ; Q)$ nous permettant de caractériser algébriquement l'image de $\Theta$ comme la plus grande partie de $F(W ; S)$ constituée des applications de degrés bornés et stable sous l'action des opérateurs $A_{i}$, celle-ci s'identifie alors facilement à l'algèbre $\Lambda$ de B. Kostant et S. KUmAR, expliquant les principaux résultats de [12] et [13].

Abstract. - Bernstein-Gel'fand-Gel'fand operators $\mathcal{A}_{i}$ are defined over the integral $T$-equivariant cohomology $H_{T}^{*}(\mathcal{F})$ of the flag variety $\mathcal{F}=G / B$ of a KačMoody group $G$. By integration over the Schubert varieties of $\mathcal{F}$, we caracterise a family $\left\{\mathcal{L}_{w}\right\}_{w \in W}$ of $H_{T}^{*}(\cdot)$-linear forms over $H_{T}^{*}(\mathcal{F})$, base of the dual of $H_{T}^{*}(\mathcal{F})$. These canonical forms are related to the operators $\mathcal{A}_{i}$ by the equality $\mathcal{L}_{w r_{i}}=\mathcal{L}_{w} \mathcal{A}_{i}$ whenever $w r_{i}>w$, impliying the intrinsic character of the compositions $\mathcal{A}_{w}$ of the $\mathcal{A}_{i}$ 's. We show that each $\mathcal{A}_{w}$ can be obtained by integration over fibers of certains fibrations above $\mathcal{F}$.

By restriction to the sub-space $W$ of $T$-fixed points of $\mathcal{F}$, we give an injectif homomorphism $\Theta$ from $H_{T}^{*}(\mathcal{F})$ into the algebra $\mathrm{F}(\mathrm{W} ; \mathrm{Q})$ of all maps defined on $W$ with values in the fraction field $Q$ of the polynomial algebra $S=\mathbb{Z}\left[\alpha_{1}, \ldots, \alpha_{n}\right]$, where $\left\{\alpha_{1}, \ldots, \alpha_{n}\right\}$ denotes the simple root system of the Lie algebra of $G$. Explicit formulas for the localisations of the $\mathcal{L}_{w}$ 's over $F(W ; Q)$ are given. We determine also the

$\left(^{*}\right)$ Texte reçu le 29 septembre 1987 , révisé le 27 mai 1988 .

A. ArAbia, Université Paris VII, UER de Mathématiques, Tour $45-55,5^{\mathrm{e}}$ étage, 2 Place Jussieu, 75251 Paris Cedex 05, France.

BULLETIN DE LA SOCIÉTÉ MATHÉMATIQUE DE FRANCE $\quad 0037-9484 / 1989 / 129 / \$ 5.00$ (C) Société mathématique de France 
localisations $A_{i}$ 's of the $\mathcal{A}_{i}$ 's over $F(W ; Q)$, which allows us to caracterise algebraically the image of $\Theta$ as the greatest subset of $F(W ; S)$ of maps of bounded degrees stable under the action of the $A_{i}$ 's, we then easily identify this image to the KOSTANT-KUMAR algebra $\Lambda$, explaining the principal results of [12] and [13].

\section{Introduction}

1.1. - Considérons une algèbre de Kač-Moody $\mathfrak{g}$ sur le corps des nombres complexes $\mathbb{C}$, notons $\mathfrak{h}$ sa sous-algèbre de Cartan, $\Pi=\left\{\alpha_{1}, \ldots, \alpha_{n}\right\}$ son système de racines simples et $W$ le groupe de Weyl correspondant muni de l'ordre de Bruhat. Soit $G$ le groupe, de sous-groupe de Borel $B$ et de tore maximal $H$, associé à $\mathfrak{g}^{\prime}=[\mathfrak{g}, \mathfrak{g}]$ et notons $K$ la forme compacte de $G$. Posons $T=K \cap H$, c'est un tore réel de dimension $n$ et d'algèbre de Lie $t$. Pour chaque $i=1,2, \ldots, n$, notons $r_{i}$ et $P^{i}$ respectivement la réflexion et le sous-groupe parabolique minimal de $G$, associés à la racine simple de même indice. Soient enfin $S=\mathbb{Z}\left[\alpha_{1}, \ldots, \alpha_{n}\right]$ l'algèbre de polynômes à coefficients entiers sur $\mathfrak{t}$ et $Q$ le corps de fractions de $S$, considérés munis de leur structure naturelle de $W$-algèbre.

1.2. - Dans cet article je m'intéresse à la cohomologie $T$-équivariante à coefficients entiers de la variété de drapeaux $\mathcal{F}=G / B=K / T$. L'outil fondamental est l'opération d'intégration sur les fibres pour une fibration $T$-équivariante $\pi: M \rightarrow N$ dont la fibre $F$ est munie d'une structure de $\mathrm{CW}$-complexe vérifiant une certaine condition d'orientabilité (cf. 2.1). Cette opération induit un homomorphisme de $H_{T}^{*}(N)$-modules noté $\tilde{\pi}_{*}$, défini sur $H_{T}^{*}(M)$ et à valeurs dans $H_{T}^{*-\operatorname{dim}(F)}(N)$. Je l'utilise pour définir les opérateurs $\mathcal{A}_{i}$ de Bernstein-Gel'fand-Gel'fand (BGG) sur $H_{T}^{*}(\mathcal{F})$ en généralisant, au contexte équivariant présent, le point de vue considéré dans [11] par V. KaČ et D. Peterson. Ceci consiste, pour chaque $i=1,2, \ldots, n$, à intégrer sur la fibre $P^{i} / B$ de la fibration $T$-équivariante $\pi_{i}: \mathcal{F} \rightarrow G / P^{i}:=\mathcal{F}^{i}$, obtenant l'homomorphisme $\tilde{\pi}_{i *}: H_{T}^{*}(\mathcal{F}) \rightarrow H_{T}^{*-2}\left(\mathcal{F}^{i}\right)$, et à considérer ensuite l'image réciproque en cohomologie équivariante $\tilde{\pi}_{i}^{*}$ induite par $\pi_{i}$ (cf. 2.1), c'est-à-dire on pose $\mathcal{A}_{i}=\tilde{\pi}_{i}^{*} \circ \tilde{\pi}_{i *}$. Le même procédé d'intégration sur les fibres est utilisé pour définir une famille de formes $H_{T}^{*}(\cdot)$-linéaires sur $H_{T}^{*}(\mathcal{F})$ que je note $\left\{\mathcal{L}_{w}\right\}_{w \in W}$, provenant des fibrations $T$-équivariantes triviales $\overline{\mathcal{F}}_{w} \rightarrow\{\cdot\}$, où $\overline{\mathcal{F}}_{w}$ dénote la variété de Schubert de $\mathcal{F}$ associée à l'élément $w$ de $W$. Dans 2.5 , je prouve que cette famille réalise une base du dual de $H_{T}^{*}(\mathcal{F})$ en tant que $H_{T}^{*}(\cdot)$-module gradué et outre le fait qu'elle détermine une vase canonique de $H_{T}^{*}(\mathcal{F})$, son intérêt principal réside dans la relation qui lie les $\mathcal{L}_{w}$ aux opérateurs $\mathcal{A}_{i}$. A ce sujet, je prouve dans la section 3 
l'affirmation suivante :

Proposition 3.2.1. - Soient $w$ un élément de $W$ et $r_{i}$ une réflexion simple, vérifiant la relation $w r_{i}>w$. On a $\mathcal{L}_{u^{\prime} r_{i}}=\mathcal{L}_{w} \circ \mathcal{A}_{i}$.

La technique de localisation permet alors d'apporter des renseignements très précis sur les propriétés algébriques de ces objets. Dans le paragraphe 2.6 j'étudie le morphisme de restriction $\Theta$ de la cohomologie $T$-équivariante de $\mathcal{F}$ à celle du sous-espace des points fixes de $T$ dans $\mathcal{F}$. Ce sous-espace s'identifie canoniquement au groupe de Weyl $W$ obtenant ainsi un homomorphisme d'algèbres $\Theta: H_{T}^{*}(\mathcal{F}) \rightarrow H_{T}^{*}(W) \equiv F_{b}(W ; S)$, où $F_{b}(W ; S)$ dénote l'algèbre des applications définies sur $W$ à valeurs dans $S$, de degrés bornés. Cet homomorphisme est injectif ( $c f$. ProposiTION 2.6.1) et permet de projeter sur l'algèbre $F(W ; Q)$ de toutes les applications de $W$ dans $Q$, les opérateurs $\mathcal{A}_{i}$ et les applications $\mathcal{L}_{w}$ (procédé de localisation). Je décris la localisation des $\mathcal{A}_{i}$ dans le théorème suivant :

Théorè.le 3.3.1. - Pour chaque $i=1,2, \ldots, n$, notons $A_{i}$ l'endomorphisme de $F\left(W^{\circ} ; Q\right)$ défini par

$$
A_{i}(f)(w)=\frac{f\left(w r_{i}\right)-f(w)}{w\left(\alpha_{i}\right)},
$$

pour tous $f \in F(W ; Q)$ et $w \in W$. On a $\Theta \circ \mathcal{A}_{i}=A_{i} \circ \Theta$.

Je retrouve ainsi les opérateurs $A_{i}$ de Kostant-Kumar, considérés pour la première fois dans leur note [12]. Cette proposition montre qu'il s'agit bien d'opérateurs de BGG dans un contexte équivariant.

Les localisations $L_{w}$ des $\mathcal{L}_{u}$, permettent de prouver très simplement le caractère intrinsèque des composées $A_{i_{1}} \circ \cdots \circ A_{i_{f}}$. Considérons à cet effet l'action du groupe de Weyl sur $F(W ; Q)$ définie par $(w \cdot f)(u)=$ $w\left(f\left(w^{-1} u\right)\right)$ pour tous $w, u \in W$ et $f \in F(W ; Q)$. On a alors :

Proposition 3.4.1. - Soient $r_{i_{1}}, \ldots, r_{i_{r}}$ des réflexions simples et posons $w=r_{i_{1}} \cdots r_{i_{\ell}}$. L'opérateur $A_{i_{1}} \circ \cdots \circ A_{i_{\text {}}}$ est un endomorphisme de $W$-module de $F(W ; Q)$ satisfaisant à l'une des conditions suivantes :

(1) si $\lg (w)=\ell$, l'application $L_{u}$ détermine entièrement l'opérateur $A_{i_{1}} \circ \cdots \circ A_{i}$ par l'égalité

$$
\left(A_{i_{1}} \circ \cdots \circ A_{i_{\vartheta}}\right)(f)(v)=v\left(L_{u} \cdot\left(v^{-1} \cdot f\right)\right),
$$

pour tous $f \in F(W ; Q)$ et $v \in W$;

(2) si $\lg (w)<\ell$, on a $A_{i_{1}} \circ \cdots \circ A_{i_{\ell}}=0$.

Notons $A_{w}=A_{i_{1}} \circ \cdots \circ A_{i_{\ell}}$ l'opérateur obtenu à partir d'une décomposition réduite $\left(r_{i_{1}}, \ldots, r_{i_{\ell}}\right)$ de $w$. La famille d'opérateurs $\left\{A_{w}\right\}_{w \in W}$ permet de décrire algébriquement l'image de la restriction $\Theta$ par le 
THÉORÈmE 3.5.1. - L'homomorphisme de restriction $\Theta: H_{T}^{*}(\mathcal{F}) \rightarrow$ $F_{b}(W ; S)$ établit un isomorphisme entre $H_{T}^{*}(\mathcal{F})$ et l'ensemble des applications $f$ de $F_{b}(W ; S)$ satisfaisant à l'une quelconque des deux conditions suivantes :

(1) $A_{w}(f)(e) \in S$, pour tout $w$ dans $W$, où e dénote l'élément neutre de $W$;

(2) $A_{w}(f) \in F_{b}(W ; S)$, pour tout $w$ dans $W$.

En particulier l'image de $\Theta$ est la plus grande partie de $F_{b}(W ; S)$ stable sous l'action des $A_{i}$.

Or c'est justement par la condition (1) que B. Kostant et S. Kumar définissent leur algèbre $\Lambda$ ( $c f$. [12], [13]) en tant que sous-algèbre de $F_{b}(W ; S)$; celle-ci s'identifie donc à la cohomologie $T$-équivariante de $\mathcal{F}$. Les principaux résultats de [13], rassemblés ici dans le ThÉorème 4.2.1 découlent alors facilement.

1.3. - Dans ce travail on trouvera aussi une interprétation cohomologique des compositions des opérateurs $\mathcal{A}_{i}$ qui suit de près des idées de M. Demazure.

Faisons agir tout élément $g$ de $G$ sur le produit $\mathcal{F} \times \mathcal{F}$ par $g(x, y)=$ $(g x, g y)$ et notons pour chaque $w \in W, \mathcal{T}_{w}$ la saturation du produit des variétés de Schubert $\overline{\mathcal{F}}_{e} \times \overline{\mathcal{F}}_{w}$ dans $\mathcal{F} \times \mathcal{F}$ par cette action de $G$. Soient $p_{1}$ et $p_{2}$ les projections de $\mathcal{F} \times \mathcal{F}$ sur $\mathcal{F}$ définies par $p_{1}(x, y)=x$ et $p_{2}(x, y)=y$, la restriction de $p_{1}$ à $\mathcal{T}_{w}$ définit une fibration $G$-équivariante de fibre $\overline{\mathcal{F}}_{w}$ et de base $\mathcal{F}$. En restreignant $p_{2}$ à $\mathcal{T}_{w}$, on a le diagramme de morphismes G-équivariants

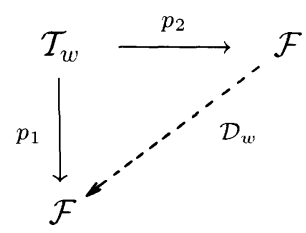

où la flèche en pointillé dénote, et dénotera tout au long de cet article, un homomorphisme entre les cohomologies équivariantes des espaces concernés; dans le cas présent $\mathcal{D}_{w}$ est l'endomorphisme de $H_{T}^{*}(\mathcal{F})$ défini $\operatorname{par} \mathcal{D}_{w}=\tilde{p}_{1 *} \circ \tilde{p}_{2}^{*}$. On a :

Proposition 3.7.1. - Soit $w$ un élément de $W$ et $\left(r_{i_{1}}, \ldots, r_{i_{\ell}}\right)$ une décomposition réduite de $w$, alors $\mathcal{D}_{w}=\mathcal{A}_{i_{1}} \circ \cdots \circ \mathcal{A}_{i_{\ell}}$. 
1.4. - Je décris sommairement maintenant le contenu des différentes sections. Dans la section 2, on s'intéresse aux CW-complexes équivariants sous l'action d'un groupe de Lie compact et ne possédant pas de cellule de dimension impaire ( $c f .2 .4$ ), ce qui fournit des résultats assez précis concernant les variétés de drapeaux $\mathcal{F}$ et $\mathcal{F}^{i}$. J'étudie les formes $\mathcal{L}_{w}$ et l'homomorphisme de restriction $\Theta$. La Proposition 2.6.1 constitue le résultat essentiel de cette section.

Dans la section 3, la plus importante de cet article, j'applique les considérations précédentes aux variétés de drapeaux $\mathcal{F}$ et $\mathcal{F}^{i}$, j'introduis les opérateurs $\mathcal{A}_{i}$ de BGG et étudie leur localisation $A_{i}$ sur $F(W ; Q)$ dans le ThÉorème 3.3.1. Je décris le caractère intrinsèque des $A_{w}$ et caractérise l'image de la restriction $\Theta$ dans le ThÉorème 3.5.1. Enfin, je définis les opérateurs $\mathcal{D}_{w}$ et prouve dans la Proposition 3.7.1 leurs rapports avec les $\mathcal{A}_{i}$.

La section 4 est destinée à montrer comment nos considérations strictement topologiques expliquent les résultats les plus importants ainsi que certains objets présents dans la note [12] de B. Kostant et S. Kumar. Ceci constitue le ThÉorème 4.2.1 qui clos ce travail.

Enfin la section 5 est un appendice destiné à rappeler la notion d'intégration sur les fibres pour une fibration à base non nécessairement de dimension finie. On y trouvera les démonstrations des assertions les plus remarquables concernant cette opération, utilisées souvent sans références tout au long des sections précédentes.

Remerciements. - Ils s'adressent à Michel Demazure pour m'avoir mis au courant des espaces $\mathcal{T}_{w}$, à Shrawan Kumar qui m'a indiqué comment simplifier la démonstration de la Proposition 2.4.1, à Jean LANNES et Michèle VERGNE pour de nombreux entretiens.

Les résultats de cet article ont été annoncés dans la note [3].

\section{Préliminaires topologiques}

2.1. Intégration sur les fibres en cohomologie équivariante. Soit $T$ un groupe de Lie compact, connexe et (dans cette section et dans l'appendice seulement) non nécessairement commutatif, agissant sur des espaces topologiques $M$ et $N$, notons $\mathbb{E} T$ le fibré universel de $T$ et $\mathbb{E} T \times{ }^{T} M$ l'espace topologique obtenu en quotientant $\mathbb{E} T \times M$ par l'action de $T$ définie par $t(p, m)=\left(p t^{-1}, t m\right)$, pour tous $p \in \mathbb{E} T$, $m \in M$ et $t \in T$ (mutatis mutandis pour $N$ ), dénotons enfin par $[p, m]$ la 
classe d'équivalence associée au couple $(p, m)$. Nous appellerons, suivant A. Borel, cohomologie $T$-équivariante de $M$ à coefficients dans un $\mathbb{Z}$ module $A$ et noterons $H_{T}^{*}(M ; A)$ la cohomologie singulière de l'espace $\mathbb{E} T \times{ }^{T} M$ à coefficients dans $A$. C'est-à-dire, on pose

$$
H_{T}^{*}(M ; A):=H^{*}\left(\mathbb{E} T \times^{T} M ; A\right) .
$$

Dans la suite lorsque aucune indication du module des coefficients ne sera donnée, on sous-entendra l'anneau $\mathbb{Z}$ des entiers relatifs.

Soit $\pi: M \rightarrow N$ une fibration $T$-équivariante orientable ( $c f$. [14]) de fibre $F$ de dimension $d$. En posant $\tilde{\pi}([p, m])=[p, \pi(m)]$, on obtient une nouvelle fibration orientable

$$
\tilde{\pi}: \mathbb{E} T \times{ }^{T} M \longrightarrow \mathbb{E} T \times{ }^{T} N
$$

de même fibre $F$. Supposons $F$ munie d'une classe d'homologie de dimension $d$. Les conditions d'orientabilité considérées ci-dessus permettent de définir, par des procédés classiques (voir 5.1), une opération cohomologique d'intégration sur les fibres

$$
\tilde{\pi}_{*}: H_{T}^{*}(M) \rightarrow H_{T}^{*}(N),
$$

homomorphisme de $H_{T}^{*}(N)$-modules gradués, de degré $-d$.

On appellera orientée une fibration orientable dont la fibre, de dimension finie, sera munie d'une classe d'homologie non triviale de dimension maximum.

Remarque. - Le cas typique auquel nous envisageons d'appliquer ces considérations est celui où la fibre $F$ s'identifie à l'une des variétés de Schubert de $G / B$ ou de $G / P^{i}$, car celles-ci admettent des structures canoniques de $\mathrm{CW}$-complexes de dimension finie ne possédant aucune cellule de codimension 1 , ce qui détermine une classe d'homologie de dimension maximum non triviale pour chacune d'elles.

2.2. - Soit maintenant $M$ un $C W$-complexe de dimension paire $d$, ne possédant pas de cellule de codimension 1 et sur lequel le groupe $T$ agit. La fibration $T$-équivariante triviale $M \rightarrow\{\cdot\}$ est clairement orientée et l'intégration sur les fibres, notée dans ce cas $\int_{M}$, est un homomorphisme de $H_{T}^{*}(\cdot)$-modules gradués

$$
\int_{M}: H_{T}^{*}(M) \longrightarrow H_{T}^{*}(\cdot)
$$

T(ME $117-1989-\mathrm{N}^{\circ} 2$ 
de degré $-d$. La proposition suivante interprète cette application dans le contexte différentiel. Supposons $M$ et $T$ munis de structures différentiables et notons $\mathbf{t}$ l'algèbre de Lie de $T$ et $\Omega_{T}^{*}(M)$ le complexe des formes différentielles $T$-équivariantes sur $M$ à coefficients complexes. Je rappelle qu'on appelle ainsi le complexe des formes différentielles $\mu(Y)$ à coefficients complexes sur $M$, dépendant polynomialement de $Y \in \mathfrak{t}$ et vérifiant une condition de $T$-équivariance évidente ( $c f .[9]$, [7], [5]). Notons aussi $S^{* / 2}\left(\mathfrak{t}^{\sim}\right)^{T}$ l'algèbre des polynômes $T$-invariants à coefficients complexes sur $\mathbf{t}$ dont la graduation habituelle a été doublée. Un résultat bien connu de A. Borel ([8]) identifie canoniquement les algèbres graduées $H_{T}^{*}(\cdot ; \mathbb{C})$

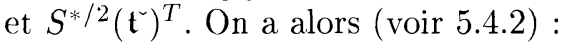

Proposition 2.2.1. - Soit $M$ une variété différentiable de dimension d, compacte connexe et orientée, munie d'une action du groupe de Lie $T$ d'algèbre de Lie $\underline{t}$. L'application qui associe à chaque forme différentielle équivariante $\mu(Y)$ de $\Omega_{T}^{*}(M)$, le polynôme $\int_{M} \mu(Y)$ (ò̀ le symbole $\int_{M}$ dénote l'intégration ordinaire) induit en cohomologie un homomorphisme de $S^{* / 2}\left(\mathfrak{t}^{`}\right)$-modules gradués qui coïncide avec l'opération d'intégration sur les fibres associée à la fibration $T$-équivariante $M \rightarrow\{\cdot\}$, moyennant l'identification canonique de $H_{T}^{*}(\cdot ; \mathbb{C})$ et $S^{* / 2}\left(\mathfrak{t}^{2}\right)^{T}$.

2.3. Évaluation à l'origine. - C'est par ce nom que nous ferons référence dans la suite à l'homomorphisme $v_{0}(M)$ (ou $v_{0}$ si l'indication de l'espace n'est pas indispensable) défini sur $H_{T}^{*}(M)$ et à valeurs dans $H^{*}(M)$, induit par la projection canonique $\mathbb{E} T \times M \rightarrow \mathbb{E} T \times{ }^{T} M$. Des arguments élémentaires prouvent la commutativité des opérations d'intégration sur les fibres et d'évaluation à l'origine (voir 5.5). Cela signifie que si l'on se donne une fibration orientée $\pi: M \rightarrow N$ et si l'on note $\tilde{\pi}$ l'application induite sur $\mathbb{E} T \times{ }^{T} M$ et à valeurs dans $\mathbb{E} T \times{ }^{T} N$ (cf. 2.1), le diagramme suivant est commutatif :

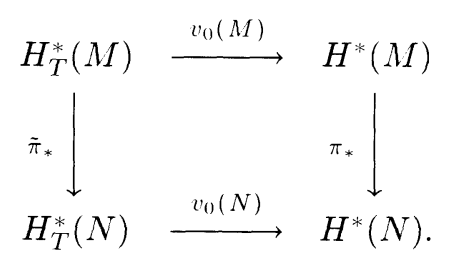

Autrement dit $v_{0}(N) \circ \tilde{\pi}_{*}=\pi_{*} \circ v_{0}(M)$.

2.4. CW-complexes T-équivariants. - A partir de cette section nous supposerons le groupe de Lie $T$ sans torsion cohomologique. 
Rappelons que, dans ce cas, A. Borel à montré ( $c f$. [8]) que la cohomologie entière paire du classifiant $\mathbb{B} T$ de $T$ est une algèbre de polynômes à coefficients entiers, à un nombre fini de variables et que sa cohomologie impaire est nulle.

Soit $M$ un CW-complexe (non nécessairement fini) ne comportant aucune cellule de dimension impaire et seulement un nombre fini de cellules en chaque dimension paire. Notons $W$ l'ensemble d'indices qui paramétrisent la famille des cellules ouvertes (paires) de $M$ notée $\left\{M_{w}\right\}_{w \in W}$. On rappelle que, pour chaque $w \in W$, l'adhérence topologique $\bar{M}_{w}$ de $M_{w}$ dans $M$ est compacte. Définissons la longueur d'un élément $w$ de $W$ (que nous notons $\lg (w))$ par $\lg (w)=\frac{1}{2} \operatorname{dim}\left(M_{w}\right)$.

Appelons $C W$-complexe $T$-équivariant la donnée d'un $C W$-complexe muni d'une action continue d'un groupe $T$ à gauche stabilisant tout sousCW-complexe (il revient au même de demander la stabilité de chaque cellule ouverte). Dans la suite nous supposerons notre $\mathrm{CW}$-complexe $\mathrm{M}$, $T$-équivariant.

Proposition 2.4.1. - Sous les hypothèses des paragraphes précédents, on a les résultats suivants :

(1) la différentielle $d_{r}$ de la suite spectrale de Leray-Serre de la fibration $\mathbb{E} T \times{ }^{T} M \rightarrow \mathbb{B} T$ est nulle pour tout $r \geq 2$;

(2) l'homomorphisme d'évaluation à l'origine $v_{0}: H_{T}^{*}(M) \rightarrow H^{*}(M)$ est surjectif ;

(3) il existe un isomorphisme $\zeta$ (non canonique) de $H_{T}^{*}(\cdot)$-modules gradués entre $H_{T}^{*}(M)$ et le produit tensoriel $H_{T}^{*}(\cdot) \otimes H^{*}(M)$ rendant commutatif le diagramme suivant :

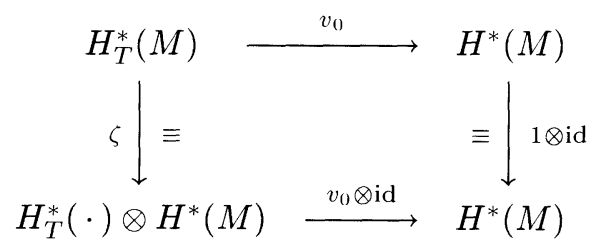

La démonstration de cette proposition est essentiellement élémentaire. Les affirmations (1) et (2) sont une conséquence immédiate du fait que $M$ ne possède pas de cellule de dimension impaire et, en ce qui concerne (3), on y reconnaît le Théorème de LERAY-Hirsch ( $c f$. [14]).

2.5. Applications $\mathcal{L}_{w}$. - L'inexistence de cellules de dimension impaire assure, que pour chaque $w \in W$, le sous-CW-complexe $\bar{M}_{w}$, 
adhérence topologique de la cellule $M_{w}$ dans $M$, est canoniquement orienté. Ce sous-CW-complexe étant d'autre part stabilisé par l'action de $T$, nous disposerons de l'homomorphisme d'intégration sur les fibres $\int_{\bar{M}_{w}}$ en cohomologie équivariante qui nous conduit à introduire les formes $\mathcal{L}_{w}$ de $H_{T}^{*}(M)$ à valeurs dans $H_{T}^{*-2 \lg (w)}(\cdot)$, en composant les applications $H_{T}^{*}(M) \stackrel{\tilde{l}_{w}^{*}}{\longrightarrow} H_{T}^{*}\left(\bar{M}_{w}\right) \stackrel{\int_{\bar{M}_{w}}}{\longrightarrow} H_{T}^{*-2 \lg (w)}(\cdot)$,

où $i_{w}: \bar{M}_{w} \rightarrow M$ dénote l'injection canonique. L'application $\mathcal{L}_{w}$ est une forme $H_{T}^{*}(\cdot)$-linéaire.

Proposition 2.5.1. - Soit $\left(\mu^{w}\right)_{w \in W}$ la base de $H^{*}(M)$ déterminée par la structure cellulaire de $M$. Il existe une base $\left(\tilde{\mu}^{w}\right)_{w \in W}$ de $H_{T}^{*}(M)$ en tant que $H_{T}^{*}(\cdot)$-module, canoniquement déterminée par les conditions suivantes :

(1) $\mathcal{L}_{u}\left(\tilde{\mu}^{w}\right)=\delta_{u}^{w}$, pour tous $u, w \in W$ (delta de Kronecker);

(2) $v_{0}\left(\tilde{\mu}^{w}\right)=\mu^{w}$, pour tout $w \in W$.

En particulier, nous avons pour tout $\mu \in H_{T}^{*}(M)$ :

(3) $\mu=\sum_{w \in W} \mathcal{L}_{w}(\mu) \tilde{\mu}^{w}$.

Démonstration. - Considérons une base d'éléments homogènes de $H_{T}^{*}(M)$ donnée par l'isomorphisme $\zeta$ de la Proposition 2.4.1, notonsla $\left(1 \otimes \mu^{w}\right)_{w \in W}$. Ses éléments satisfont aux égalités suivantes :

(a) $\mathcal{L}_{v}\left(1 \otimes \mu^{w}\right)=0$, si $\lg (w)<\lg (v)$;

(b) $\mathcal{L}_{v}\left(1 \otimes \mu^{w}\right)=\delta_{v}^{w}$, si $\lg (v)=\lg (w)$.

En effet, l'égalité (a) découle de la relation d'appartenance évidente $\mathcal{L}_{v}\left(1 \otimes \mu^{w}\right) \in H^{2(\lg (w)-\lg (v))}(\mathbb{B} T)$. Pour (b), on remarque que, puisque $\mathcal{L}_{v}\left(1 \otimes \mu^{w}\right)$ est de degré nul, on aura (cf. 2.3) les égalités

$$
\mathcal{L}_{v}\left(1 \otimes \mu^{w}\right)=v_{0} \mathcal{L}_{v}\left(1 \otimes \mu^{w}\right)=\int_{\bar{M}_{v}} v_{0}\left(1 \otimes \mu^{w}\right)=\int_{\bar{M}_{v}} \mu^{w}=\delta_{v}^{w}
$$

Lorsque $\lg (v)<\lg (w)$, rien ne peut être dit en général de $\mathcal{L}_{v}\left(1 \otimes \mu^{w}\right)$. Nous allons donc redresser la base des $1 \otimes \mu^{w}$ en appliquant le procédé de Gram-Schmidt. Soit $m$ un entier positif et supposons avoir défini $\tilde{\mu}^{u}$ pour $\lg (u)<m$ satisfaisant aux conditions (1) et (2). Posons, pour chaque $w$ de longueur $m$,

$$
\tilde{\mu}^{w}=1 \otimes \mu^{w}-\sum \mathcal{L}_{u}\left(1 \otimes \mu^{w}\right) \tilde{\mu}^{u},
$$

où la sommation est indexée par les $u \in W$ vérifiant $\lg (u)<\lg (w)$. La condition (1) est alors automatiquement vérifiée par $\tilde{\mu}^{w}$ et la condition (2) 
résulte du fait que $\mathcal{L}_{u}\left(1 \otimes \mu^{w^{\prime}}\right) \in H^{2\left(\lg \left(w^{\prime}\right)-\lg (u)\right)}(\mathbb{B} T)$ et donc appartient au noyau de $v_{0}$.

2.6. Restriction aux points fixes. - Notons $M^{T}$ le sous-espace des points fixes de $T$ dans $M$, nous rappelons que, puisque $\mathbb{E} T \times^{T} M^{T} \equiv$ $\mathbb{B} T \times M^{T}$, on a un isomorphisme d'algèbres entre $H_{T}^{*}\left(M^{T}\right)$ et $H_{T}^{*}(\cdot) \otimes$ $H^{*}\left(M^{T}\right)$. D'autre part, l'inclusion canonique $\mathbb{B} T \times M^{T} \rightarrow \mathbb{E} T \times{ }^{T} M$ induit l'homomorphisme de restriction $\Theta$ en cohomologie équivariante

$$
\Theta: H_{T}^{*}(M) \longrightarrow H_{T}^{*}(\cdot) \otimes H^{*}\left(M^{T}\right) .
$$

Remarque. - On pourrait penser que $\Theta$, modulo l'isomorphisme canonique entre $H_{T}^{*}(M)$ et $H_{T}^{*}(\cdot) \otimes H^{*}(M)$ évoqué par la proposition précédente, n'est autre que l'homomorphisme de restriction aux points fixes id $\otimes i\left(M^{T}\right)^{*}$, où $i\left(M^{T}\right)$ dénote l'inclusion de $M^{T}$ dans $M$. En réalité ce dernier s'identifie à $\Theta$ seulement sur les gradués des algèbres en question, relativement à la filtration par squelettes de $M$; il aura en ce sens un noyau non trivial en général, alors que nous prouverons dans la proposition suivante l'injectivité de $\Theta$.

Proposition 2.6.1. Soit $M^{T}$ l'ensemble des points fixes de $T$ dans $M$.

(1) L'homomorphisme d'algèbres $\Theta: H_{T}^{*}(M) \rightarrow H_{T}^{*}(\cdot) \otimes H^{*}\left(M^{T}\right)$ est injectif.

(2) Notons $Q$ le corps de fractions de $H_{T}^{*}(\cdot)$. Pour chaque élément $w$ de $W$, il existe un homomorphisme canonique

$$
L_{u}: H_{T}^{*}(\cdot) \otimes H^{*}\left(M^{T}\right) \rightarrow Q
$$

de $H_{T}^{*}(\cdot)$-modules satisfaisant à l'égalité $\mathcal{L}_{u}=L_{w}$ ○ $\Theta$.

(3) Si $M^{T}$ est discret l'image de $\Theta$ est constituée des éléments $\nu \in$ $H_{T}^{*}(\cdot) \otimes H^{*}\left(M^{T}\right)$ tels que $L_{w}(\nu) \in H_{T}^{*}(\cdot)$ pour tout $w \in W$.

Démonstration. - Pour chaque entier positif $m$ notons $M_{m}$ le $m$ squelette de $M$ et $\Theta_{m}$ l'homomorphisme de restriction de $H_{T}^{*}\left(M_{m}\right)$ à $H_{T}^{*}\left(M_{m}^{T}\right)$ et considérons le diagramme commutatif $(\mathrm{D})$ de la page suivante, où les produits tensoriels sont faits sur $H_{T}^{*}(\cdot)$ et où $\alpha$ est induite par les restrictions de $H_{T}^{*}(M)$ aux $H_{T}^{*}\left(M_{m}\right)$ et $\beta$ par les applications

$$
\mathrm{id} \otimes 1: H_{T}^{*}\left(M_{m}\right) \longrightarrow H_{T}^{*}\left(M_{m}\right) \otimes Q
$$

(mutatis mutandis pour $\alpha^{T}$ et $\beta^{T}$ ), enfin $\Theta_{\infty}$ et $\Theta_{\infty} \otimes$ id sont des notations T(ME $117 \cdots 1989-\mathrm{N}^{\mathrm{O}} 2$ 
abrégées pour $\varliminf_{m}\left(\Theta_{m}\right)$ et $\varliminf_{m}\left(\Theta_{m} \otimes \mathrm{id}\right)$ respectivement.

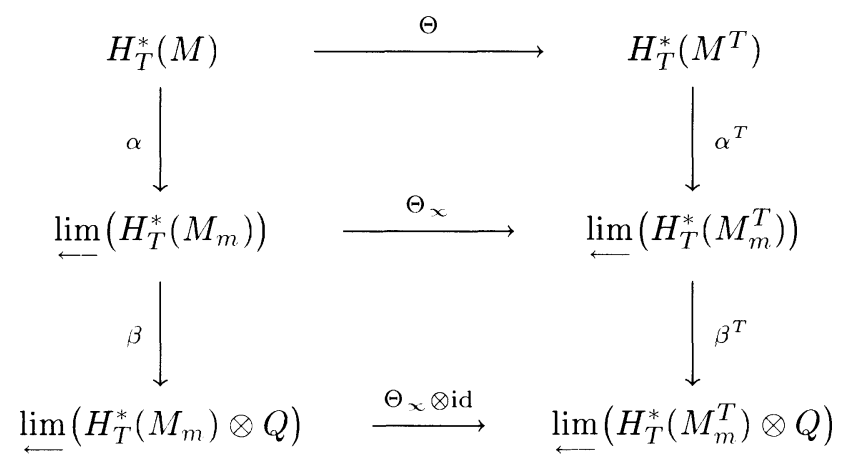

Diagramme (D)

La compacité de $M_{m}$ permet d'appliquer le théorème de localisation d'Atiyah-Segal qui affirme que $\Theta_{m} \otimes$ id établit un isomorphisme entre $H_{T}^{*}\left(M_{m}\right) \otimes Q$ et $H_{T}^{*}\left(M_{m}^{T}\right) \otimes Q$, impliquant que la limite $\Theta_{\infty} \otimes$ id est aussi bijective. D'autre part, on sait que $\alpha$ est une injection de même que $\beta$, puisque $H_{T}^{*}\left(M_{m}\right)$ est sans torsion. Ces premières remarques prouvent déjà l'injectivité de $\Theta$ ainsi que celle de chaque $\Theta_{m}$ et donc celle de $\Theta_{\infty}$. Soit maintenant $w$ un élément de $W$. La forme linéaire $\mathcal{L}_{w}$ définit une application du système projectif $\left\{H_{T}^{*}\left(M_{m}\right) \otimes Q\right\}_{m \geq 2 \lg (w)}$ à valeurs dans $Q$. Notons $\mathcal{L}_{w} \otimes 1$ la forme correspondant à la limite projective $\varliminf_{T}\left(H_{T}^{*}\left(M_{m}\right) \otimes Q\right)$. L'application annoncée dans (2) est alors obtenue en posant

$$
L_{w}=\left(\mathcal{L}_{w} \otimes 1\right) \circ\left(\Theta_{\infty} \otimes \mathrm{id}\right)^{-1} \circ \beta^{T} \circ \alpha^{T} .
$$

Supposons maintenant $M^{T}$ discret et prouvons (3). La condition étant clairement nécéssaire montrons sa suffisance. Donnons-nous donc un élément $\nu$ de $H_{T}^{*}\left(M^{T}\right)$ vérifiant la condition en question. L'élément $\alpha^{T}(\nu)$ se relève en un système projectif dont le $m$-ième terme $\alpha_{m}^{T}(\nu)$ appartient à $H_{T}^{*}\left(M_{m}^{T}\right)$.

Pour chaque entier positif $m$, considérons le terme d'indice $m$ du diagramme (D) (voir page suivante) et posons $\mu_{m}=\sum L_{w}(\nu) \tilde{\mu}^{w}$, où la somme est indexée par les $w$ de $W$ satisfaisant à l'inégalité $2 \lg (w) \leq m$. S'agissant d'une somme finie à coefficients dans $H_{T}^{*}(\cdot)$ et concernant les éléments de la base canonique de $H_{T}^{*}\left(M_{m}\right)$, elle détermine un élément $\mu_{m}$ de $H_{T}^{*}\left(M_{m}\right)$ vérifiant, par construction, l'égalité

$$
\mathcal{L}_{w}\left(\mu_{m}\right)=L_{w}(\nu)=L_{w}\left(\alpha_{m}^{T}(\nu)\right) .
$$




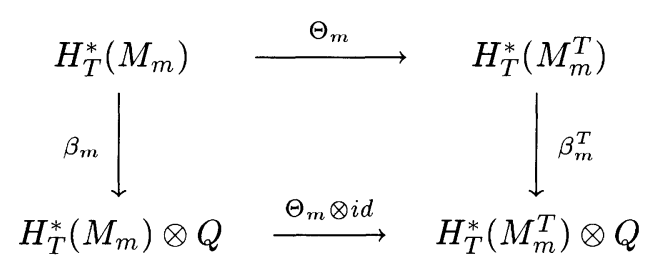

Par conséquent, on a

$$
\left(\left(\mathcal{L}_{w} \otimes 1\right) \circ(\Theta \otimes \mathrm{id})^{-1} \circ \beta_{m}^{T}\right)\left(\Theta\left(\mu_{m}\right)-\alpha_{m}^{T}(\nu)\right)=0
$$

pour tout $w$ vérifiant $2 \lg (w) \leq m$. On conclut alors, par l'injectivité de $\beta_{m}^{T}$ (puisque $M_{m}^{T}$ est discret), que $\Theta_{m}\left(\mu_{m}\right)=\alpha_{m}^{T}(\nu)$, et, par l'injectivité de $\Theta_{m}$, que le degré cohomologique de $\mu_{m}$ est majoré par celui de $\nu$. Ceci étant, en notant $j_{m}: M_{m-1} \rightarrow M_{m}$ et $j_{m}^{T}: M_{m-1}^{T} \rightarrow M_{m}^{T}$ les injections canoniques, nous avons les égalités

$$
\Theta \circ j_{m}^{*}\left(\mu_{m}\right)=\left(j_{m}^{T}\right)^{*} \circ \Theta\left(\mu_{m}\right)=\left(j_{m}^{T}\right)^{*} \circ \alpha_{m}^{T}(\nu)=\alpha_{m-1}^{T}(\nu)=\Theta\left(\mu_{m-1}\right)
$$

qui prouvent, grâce à l'injectivité de $\Theta$, que $j_{m}^{*}\left(\mu_{m}\right)=\mu_{m-1}$; la famille $\left\{\mu_{m}, j_{m}^{*}\right\}_{m \geq 0}$ est donc projective. Enfin, il existe bien un élément $\mu \in$ $H_{T}^{*}(M)$ dont les restrictions aux $M_{m}$ égalent les $\mu_{m}$ puisque les degrés cohomologiques des $\mu_{m}$ sont majorés par celui de $\nu$. L'injectivité de $\alpha^{T}$ permet alors de conclure à l'égalité $\Theta(\mu)=\nu$, terminant la démonstration de la proposition.

Remarque. - Les applications $L_{w}$ ont été définies sur la limite projective $\lim _{(}\left(H_{T}^{*}\left(M_{m}^{T}\right) \otimes Q\right)$ qui jouera à partir de la section suivante un rôle suffisament important pour que nous lui donnions une interprétation en des termes plus simples. Lorsque $M^{T}$ est discret l'algèbre $H_{T}^{*}\left(M_{m}^{T}\right) \equiv H_{T}^{*}(\cdot) \otimes H^{0}\left(M_{m}^{T}\right)$ est canoniquement isomorphe à l'algèbre $F_{b}\left(M_{m}^{T} ; H_{T}^{*}(\cdot)\right)$ des applications de $M_{m}^{T}$ à valeurs dans $H_{T}^{*}(\cdot)$ de degrés bornés (la somme et le produit étant définis point par point) et ceci pour tout $0 \leq m \leq \infty$. Lorsque $m<\infty$ la condition de degrés bornés est superflue et $\varliminf_{(}\left(H_{T}^{*}\left(M_{m}^{T}\right) \otimes Q\right)$ s'identifie à l'algèbre $F\left(M^{T} ; Q\right)$ de toutes les applications de $M^{T}$ à valeurs dans $Q$. La composée $\beta^{T} \circ \alpha^{T}$ s'interprète par conséquent comme l'injection canonique de $F_{b}\left(M^{T} ; H_{T}^{*}(\cdot)\right)$ dans $F\left(M^{T} ; Q\right)$. 


\section{Cohomologie T-équivariante des variétés de drapeaux $G / B$}

3.1. Notations et rappels. - Pour tous les détails concernant ce premier paragraphe nous renvoyons à l'article de V. Kač [10]. Soit $\mathfrak{g}$ une algèbre de Kač-Moody sur le corps des nombres complexes $\mathbb{C}$, de sousalgèbre de Cartan $\mathfrak{h}$, système de racines simples $\Pi=\left\{\alpha_{1}, \ldots, \alpha_{n}\right\}$ et groupe de Weyl $W$ qu'on supposera muni de l'ordre de Bruhat. Notons $G$ le groupe, de sous-groupe de Borel $B$ et tore maximal $H$, associé à $\mathfrak{g}^{\prime}=[\mathfrak{g}, \mathfrak{g}]$. Pour chaque $i=1,2, \ldots, n$, notons $r_{i}$ et $P^{i}$ respectivement la réflexion simple et le sous-groupe parabolique minimal de $G$, associés à la racine $\alpha_{i}$, le groupe de Weyl de $\left(P^{i}, B\right)$ s'identifie à $\left\{1, r_{i}\right\}$. Soit $K$ la forme compacte de $G$ et posons $T=K \cap H$. Le sous-groupe $T$ est un tore réel de dimension $n$ et d'algèbre de Lie $\mathfrak{t}$. L'espace classifiant $\mathbb{B} T$ de $T$, peut être construit comme limite inductive des produits de $n$ copies de l'espace projectif $\mathbb{P}^{m}(\mathbb{C})$ lorsque $m$ tend vers l'infini, ce qui prouve immédiatement que $H^{*}(\mathbb{B} T)$ est isomorphe à l'algèbre graduée $\mathbb{Z}^{* / 2}\left[X_{1}, \ldots, X_{n}\right]$ où les $X_{i}$ dénotent les duaux des classes fondamentales de chaque copie de $\mathbb{P}^{1}(\mathbb{C})$ et sont donc considérés de degré 2 . Le groupe de Weyl opère par translations à droite sur $\mathbb{B} T$ (on peut prendre $\mathbb{E} T=\mathbb{E} K$ comme fibré universel) et l'identification $X_{i} \equiv \alpha_{i}$ fournit un isomorphisme de $W$-algèbres graduées entre $H^{*}(\mathbb{B} T)$ et l'algèbre $\mathbb{Z}^{* / 2}\left[\alpha_{1}, \ldots, \alpha_{n}\right]$, que nous noterons, pour abréger, $S^{*}$ et même $S$ lorsque la référence à la graduation ne sera pas essentielle. On notera $Q$ le corps de fractions de $S$.

La variété de drapeaux de $G$ est par définition l'espace topologique $\mathcal{F}=G / B=K / T$; on la considérera munie de l'action naturelle de $T$ à gauche. Nous rappelons que cette variété admet une structure de CWcomplexe dont les cellules ouvertes $\mathcal{F}_{w}$, indexées par les éléments $w \mathrm{du}$ groupe de Weyl $W$, satisfont aux conditions suivantes :

(1) $\mathcal{F}=\bigcup_{w \in W} \mathcal{F}_{w}$ et $\overline{\mathcal{F}}_{w}=\bigcup_{v \leq w} \mathcal{F}_{v}$, où $\overline{\mathcal{F}}_{w}$ dénote la fermeture topologique de $\mathcal{F}_{w}$;

(2) chaque cellule ouverte $\mathcal{F}_{w}$ est munie d'une structure d'espace vectoriel complexe et $\operatorname{dim}_{\mathbb{C}}\left(\mathcal{F}_{w}\right)=\lg (w)$, où $\lg (w)$ dénote la longueur de $w$ relativement à l'ordre de Bruhat de $W$.

(3) l'action de $T$ stabilise chaque cellule ouverte $\mathcal{F}_{w}$.

Pour chaque $1 \leq i \leq n$, posons $\mathcal{F}^{i}=G / P^{i}$. Il s'agit d'une variété de drapeaux munie d'une structure de $\mathrm{CW}$-complexe indexée par l'ensemble $W^{i}=W /\left\{1, r_{i}\right\}$, satisfaisant aux mêmes conditions (1), (2) et (3) que $G / B$, à condition bien sûr de substituer $W$ par $W^{i}$.

Les variétés $\mathcal{F}$ et $\mathcal{F}^{i}$ sont simplement connexes et les projections canoniques $\pi_{i}: \mathcal{F} \rightarrow \mathcal{F}^{i}$ sont orientables de fibres $P^{i} / B$ orientées 
canoniquement par leur structure complexe, la technique d'intégration sur les fibres du paragraphe 2.1 pourra donc être appliquée sans autre vérification.

Le fait que les structures de CW-complexe de $\mathcal{F}$ et des $\mathcal{F}^{i}$ soient $T$ équivariantes et dépourvues de cellules de dimension impaire permet aussi de leur appliquer la Proposition 2.6.1.

Nous rappelons enfin que les ensembles des points fixes de $T$ dans $\mathcal{F}$ et dans $\mathcal{F}^{i}$ s'identifient canoniquement aux groupes $W$ et $W^{i}$ respectivement. On a $H_{T}^{*}(W) \equiv F_{b}\left(W ; S^{*}\right)$ et $H_{T}^{*}\left(W^{i}\right) \equiv F_{b}\left(W^{i} ; S^{*}\right)$.

Les applications $L_{w}$ du paragraphe 2.6, se trouvent maintenant définies sur l'algèbre $F(W ; Q)$ et permettent de caractériser l'image de l'injection $\Theta: H_{T}^{*}(\mathcal{F}) \rightarrow F_{b}\left(W ; S^{*}\right)$. Le but de cette section est de les interpréter algébriquement de façon à donner une description purement algébrique de l'image en question.

3.2. Opérateurs $\mathcal{A}_{i}$ de $H_{T}^{*}(\mathcal{F})$. - Soit $\alpha_{i}$ une racine simple et considérons la fibration $T$-équivariante $\pi_{i}: \mathcal{F} \rightarrow \mathcal{F}^{i}$ de fibre la sphère $\mathbb{S}^{2} \equiv P^{i} / B$ orientée par sa structure complexe. Rappelons que, d'après le paragraphe 2.1, la fibration $\pi_{i}$ induit une fibration $\tilde{\pi}_{i}$ de base $\mathbb{E} T \times{ }^{T} \mathcal{F}^{i}$ et d'espace total $\mathbb{E} T \times{ }^{T} \mathcal{F}$ et que, par intégration sur les fibres, on a l'homomorphisme de $H_{T}^{*}\left(\mathcal{F}^{i}\right)$-modules $\tilde{\pi}_{i *}: H_{T}^{*}(\mathcal{F}) \rightarrow H_{T}^{*-2}\left(\mathcal{F}^{i}\right)$.

En nous inspirant de travaux de V. KaČ et D. Peterson, nous posons pour chaque $i=1,2, \ldots, n$

$$
\mathcal{A}_{i}=\tilde{\pi}_{i}^{*} \circ \tilde{\pi}_{i *}
$$

Ce sont des endomorphismes de $H_{T}^{*}(\mathcal{F})$ de $S^{*}$-module, de degré -2 et de carré nul.

Remarque. - La formule $(\mathcal{A})$ est utilisée par ces auteurs pour définir les endomorphismes $\mathcal{A}_{i}$ sur $H^{*}(\mathcal{F})$ (cf. [10], [11]). Il est alors facile de voir que l'évaluation à l'origine commute à ces opérateurs, ce qui permet de projeter les identités de cette section à $H^{*}(\mathcal{F})$ grâce notamment à la surjectivité de $v_{0}$.

Proposition 3.2.1. - Soient $w$ un élément de $W$ et $r_{i}$ une réflexion simple, vérifiant la relation $w r_{i}>w$. On a $\mathcal{L}_{w r_{i}}=\mathcal{L}_{w} \circ \mathcal{A}_{i}$.

Démonstration. - Considérons la restriction $\pi_{i}:: \overline{\mathcal{F}}_{w r_{i}} \rightarrow \overline{\mathcal{F}}_{[w]}^{i}$, où $[w]$ dénote la classe de $w$ dans $W^{i}$, de la projection $\pi_{i}: \mathcal{F} \rightarrow \mathcal{F}^{i}$. On a le TOME $117-1989-\mathrm{N}^{\mathrm{O}} 2$ 
diagramme commutatif

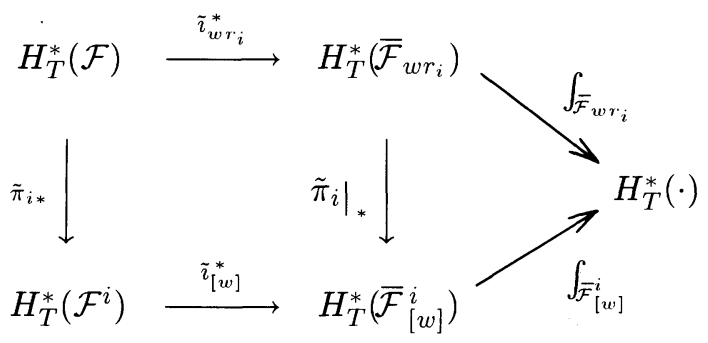

entraînant l'égalité

$$
\mathcal{L}_{w r_{i}}=\mathcal{L}_{[w]} \circ \tilde{\pi}_{i *}
$$

puisque

$$
\mathcal{L}_{w r_{i}}=\int_{\overline{\mathcal{F}}_{w r_{i}}} \circ \tilde{\imath}_{w r_{i}}^{*}=\left.\int_{\overline{\mathcal{F}}_{[w]}^{i}} \circ \tilde{\pi}_{i}\right|_{*} \circ \tilde{\imath}_{w r_{i}}^{*}=\mathcal{L}_{[w]} \circ \tilde{\pi}_{i *} .
$$

D'autre part, dans la décomposition de Bruhat de $G$ relativement à $B$, on a $B w B \cup B w r_{i} B=B[w] P^{i}$, impliquant que par la projection

$$
\pi_{i}:\left(B w B \cup B w r_{i} B\right) / B \longrightarrow B[w] P^{i} / P^{i},
$$

la classe fondamentale de $\overline{\mathcal{F}}_{[w]}^{i}$ est déterminée par la paramétrisation de la celulle $B w B / B=\mathcal{F}_{w}$ et donc que $\mathcal{L}_{[w]}=\mathcal{L}_{w} \circ \tilde{\pi}_{i}^{*}$, d'où la proposition après remplacement dans (1).

3.3. Localisation et opérateurs $A_{i}$. - Nous avons vu dans la démonstration de la Proposition 2.6.1 comment $\Theta$ induit un isomorphisme entre $\varliminf_{(}\left(H_{T}^{*}\left(\mathcal{F}_{m}\right) \otimes_{S} Q\right)$ et $F(W ; Q)$. Pour chaque $i=1,2, \ldots, n$, filtrons la fibration $\pi_{i}: \mathcal{F} \rightarrow \mathcal{F}^{i}$ par les $m$-squelettes de $\mathcal{F}^{i}$ et posons $\mathcal{F}_{m, i}=\pi_{i}^{-1}\left(\mathcal{F}_{m}^{i}\right)$; c'est la réunion des cellules ouvertes $\mathcal{F}_{w}$ où les indices $w$ dans $W$ satisfont à l'une des conditions suivantes :

(i) $2 \lg (w) \leq m$;

(ii) $2 \lg (w)=m+2$ et $w r_{i}<w$.

Par conséquent pour chaque $i=1,2, \ldots, n$, on a les inclusions $\mathcal{F}_{m+2} \supseteq$ $\mathcal{F}_{m, i} \supseteq \mathcal{F}_{m}$ de $T$-espaces, qui entraînent un isomorphisme canonique entre $\varliminf_{T}\left(H_{T}^{*}\left(\mathcal{F}_{m}\right) \otimes_{S} Q\right)$ et $\varliminf_{\longrightarrow}\left(H_{T}^{*}\left(\mathcal{F}_{m, i}\right) \otimes_{S} Q\right)$. Or, sur ce dernier, nous 
définissons par la formule $(\mathcal{A})$ du paragraphe 3.2 , l'opérateur $\mathcal{A}_{i}$ qui va se projeter par les identifications précédentes sur $F(W ; Q)$, la démonstration $\mathrm{du}$ théorème prochain en donne une interprétation que nous noterons $A_{i}$.

Théorème 3.3.1. - Pour chaque $i=1,2, \ldots, n$, notons $A_{i}$ l'endomorphisme de $F(W ; Q)$ défini par

$$
A_{i}(f)(w)=\frac{f\left(w r_{i}\right)-f(w)}{w\left(\alpha_{i}\right)},
$$

pour tous $f \in F(W ; Q)$ et $w \in W$. On a alors $h \circ \Theta \circ \mathcal{A}_{i}=A_{i} \circ h \circ \Theta$, où $h$ dénote l'injection canonique de $F_{b}\left(W ; S^{*}\right)$ dans $F(W ; Q)$.

Nota. - Ces endomorphismes ont été considérés pour la première fois dans [12] par B. Kostant et S. Kumar, nous indiquerons dans le paragraphe 3.6 le lien avec les opérateurs de BGG sur l'algèbre $S$ classiques.

Démonstration. - Considérons, pour chaque entier positif $m$, le diagramme de fibrations suivant

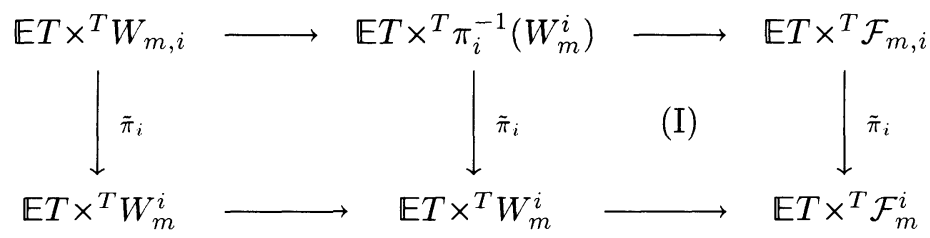

où $\mathcal{F}_{m}^{i}$ dénote le $m$-squelette de $\mathcal{F}^{i}$. Son image réciproque par $\pi_{i}$ est désignée par $\mathcal{F}_{m, i}$. Les points fixes de $T$ dans $\mathcal{F}_{m}^{i}$ constituent la partie notée $W_{m}^{i}$ et ceux de $\mathcal{F}_{m, i}$ la partie $W_{m, i}$. Ces ensembles de points fixes se décrivent sans difficultés, $W_{m, i}$ est l'ensemble des $w$ vérifiant les conditions (i) et (ii) données plus haut, alors que $W_{m}^{i}$ est la projection de $W_{m, i}$ dans $W^{i}$. Ceci étant, on remarquera que les fibrations de (I) sont de même fibre $P^{i} / B$ alors que la fibration de gauche est à fibres discrètes $\left\{1, r_{i}\right\}$. En cohomologie, on obtient le diagramme

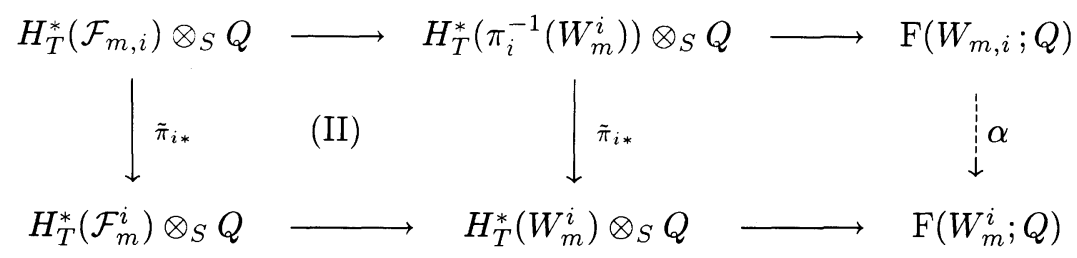

TOME $117-1989-\mathrm{N}^{\circ} 2$ 
où les flèches horizontales sont des isomorphismes et le sous-diagramme (II) est commutatif. L'intérêt de ce diagramme repose surtout en son terme central permettant une explicitation aisée de $\tilde{\pi}_{i *}$, nécessaire pour déterminer la flèche discontinue $\alpha$ rendant le diagramme commutatif.

Commençons par préciser la nature de l'ensemble $\pi_{i}^{-1}\left(W_{m}^{i}\right)$. Topologiquement il s'agit, bien sûr, du produit cartésien $W_{m}^{i} \times \mathbb{S}^{2}$ et, en ce qui concerne sa structure de $T$-espace, on remarque que chaque classe de $W_{m}^{i}$ s'écrit de manière unique comme un ensemble $\left\{w, w r_{i}\right\}$ avec $w r_{i}>w$. Notons $[w]$ une telle classe. La sphère $P^{i} / B$ s'injectant canoniquement dans $\mathcal{F}=G / B$, posons $\mathbb{S}_{w}^{2}=w\left(P^{i} / B\right) \subseteq G / B$ et considérons-la munie de l'action de $T$ restreinte de $G / B$ à $\mathbb{S}_{w}^{2}$. Nous obtenons ainsi l'égalité de $T$-espaces

$$
\pi_{i}^{-1}\left(W_{m}^{i}\right)=\coprod_{[w] \in W_{m}^{i}} \mathbb{S}_{w}^{2}
$$

L'explicitation de l'homomorphisme du terme central de notre dernier diagramme, revient ainsi à expliciter l'intégrale

$$
\int_{\mathbb{S}^{2}}: \prod_{[w] \in W_{m}^{i}} H_{T}^{*}\left(\mathbb{S}_{w}^{2}\right) \longrightarrow \prod_{[w] \in W_{m}^{i}} Q
$$

dont il suffira de comprendre $\int_{\mathbb{S}_{w}^{2}}: H_{T}^{*}\left(\mathbb{S}_{w}^{2}\right) \rightarrow Q$, pour chaque $w \in W$ vérifiant l'inégalité $w r_{i}>w$.

L'idée maintenant est de remarquer que, puisque les modules que nous considérons sont sans torsion, nous ne perdrons aucun renseignement en tensorisant par le corps des nombres complexes, avec l'avantage supplémentaire de pouvoir appliquer les techniques de géométrie différentielle, notamment l'utilisation du complexe des formes différentielles $T$-équivariantes sur $\mathbb{S}_{w}^{2}$ et l'intégration ordinaire, pour expliciter l'intégration sur les fibres ( $c f .2 .2)$. Soit donc $\mu(Y)$ un cocycle $T$-équivariant de $\mathbb{S}_{w}^{2}$. Pour évaluer $\int_{\mathbb{S}^{2}} \mu(Y)$ nous allons utiliser la formule de localisation de N. Berline et M. Vergne [6], qui, dans le cas présent, s'explicite par l'égalité

$$
\int_{\mathbb{S}_{w}^{2}} \mu(Y)=-\sqrt{-1}\left(\frac{\Theta(\mu)(w)(Y)}{\operatorname{Pf}(L(Y)(w))}+\frac{\Theta(\mu)\left(w r_{i}\right)(Y)}{\operatorname{Pf}\left(L(Y)\left(w r_{i}\right)\right)}\right),
$$

où $L(Y)(w)$ (resp. $L(Y)\left(w r_{i}\right)$ ) dénote la dérivée de Lie de l'action infinitésimale de $Y \in \mathbf{t}$ sur l'espace tangent à $\mathbb{S}_{w}^{2}$ au point fixe $w$ (resp. $w r_{i}$ ) et où $\mathrm{Pf}$ désigne le pfaffien; $\Theta$ dénote, par abus, l'homomorphisme de restriction aux points fixes

$$
\Theta: H_{T}^{*}\left(\mathbb{S}_{w}^{2}\right) \longrightarrow F\left(\left\{w, w r_{i}\right\} ; S^{*}\right) .
$$


Un calcul simple montre que le pfaffien $\operatorname{Pf}(L(Y)(w))$ vaut $\sqrt{-1} w\left(\alpha_{i}\right)(Y)$ $(c f .[2])$, ce qui après remplacement dans $(\dagger)$ entraîne l'égalité

$$
\int_{\mathbb{S}_{w}^{2}} \mu=\frac{\Theta(\mu)\left(w r_{i}\right)-\Theta(\mu)(w)}{w\left(\alpha_{i}\right)}
$$

pour toute classe de cohomologie $T$-équivariante $\mu$ sur $\mathbb{S}_{w}^{2}$. Cette formule nous donne très précisément l'interprétation de $\alpha$ cherchée. On a

$$
\alpha(f)([w])=\frac{f\left(w r_{i}\right)-f(w)}{w\left(\alpha_{i}\right)},
$$

pour tous $f \in \mathrm{F}\left(W_{m, i} ; \mathrm{S}\right)$ et $w \in W_{m, i}$ vérifiant $w r_{i}>w$. Or le fait de remplacer $w$ par $w r_{i}$ dans l'expression de droite de $(\dagger \dagger)$ ne modifie pas l'identité, partant la condition $w r_{i}>w$ est superflue et, en rappelant que $\mathcal{A}_{i}=\tilde{\pi}_{i}^{*} \circ \tilde{\pi}_{i *}$, nous obtenons, après localisation, l'égalité $A_{i}=\tilde{\pi}_{i}^{*} \circ \alpha$, c'est-à-dire

$$
A_{i}(f)(w)=\frac{f\left(w r_{i}\right)-f(w)}{w\left(\alpha_{i}\right)}
$$

pour tous $f \in F\left(W_{m, i} ; S\right)$ et $w \in W_{m, i}$. Le passage à la limite projective termine la démonstration du théorème.

Corollaire 3.3.1. - Soient $w$ un élément de $W$ et $r_{i}$ une réflexion simple vérifiant la relation $w r_{i}>w$. On a $L_{w r_{i}}=L_{w} \circ A_{i}$ sur $F(W ; Q)$.

Démonstration. - Il suffit de le vérifier sur $F\left(W_{m, i} ; S^{*}\right)$ pour $m>$ $2 \lg (w)+2$, ce qui est immédiat d'après la Proposition 3.2.1.

Remarque. - L'itération de cette égalité permet d'expliciter complètement $L_{w}$ à partir d'une décomposition réduite de $w$, cependant une autre voie de calcul est donnée par l'application de la formule de localisation de N. Berline et M. Vergne ( $c f$. [6]). C'est ainsi que j'ai procédé dans la thèse [1] et s'agissant d'un résultat non encore publié nous profitons pour l'énoncer dans cet article.

Proposition 3.3.1. - Soient $w$ et $u$ des éléments de $W$. Considérons une décomposition réduite $\left(r_{i_{1}}, \ldots, r_{i_{\ell}}\right)$ de $w$ et posons, lorsque $u \leq w$,

$$
q_{w}^{u}=\sum \prod_{j=1}^{\ell}\left(-\sigma_{1} \cdots \sigma_{j}\left(\alpha_{i_{j}}\right)\right)^{-1}
$$

où la sommation est indexée par les $\ell$-uplets $\left(\sigma_{1}, \ldots, \sigma_{\ell}\right)$ avec $\sigma_{j} \in\left\{1, r_{i_{j}}\right\}$ et $\sigma_{1} \cdots \sigma_{\ell}=u$. Les $q_{w}^{u}$ sont des fractions rationnelles de $Q$ vérifiant

TOME $117-1989-\mathrm{N}^{\circ} 2$ 
l'égalité, pour tout $\mu \in H_{T}^{*}(\mathcal{F})$,

$$
\mathcal{L}_{w}(\mu)=\sum_{u \leq w} q_{w}^{u} \Theta(\mu)(u) .
$$

Démonstration. - De même que nous l'avons fait dans la démonstration du THÉORÈmE 3.3.1, tensorisons par le corps des nombres complexes de façon à nous permettre les techniques de la géométrie différentielle. Il s'agit donc d'évaluer $\int_{\overline{\mathcal{F}}_{w}} \mu(Y)$ où $\mu(Y)$ est un cocycle $T$-équivariant sur $\mathcal{F}$. En considérant la désingularisation de Hansen $g_{w}: \Gamma_{w} \rightarrow \overline{\mathcal{F}}_{w}$ (cf. [2]) associée à la décomposition réduite $\left(r_{i_{1}}, \ldots, r_{i_{\ell}}\right)$ de $w$, nous nous ramenons à $\int_{\Gamma_{w}} \mu(Y)$. Ici, $\Gamma_{w}$ est une variété de Bott-Samelson, c'est une $T$-variété lisse avec $2^{\ell}$ points fixes, indexés par les $\ell$-uplets $\left(\sigma_{1}, \ldots, \sigma_{\ell}\right)$ avec $\sigma_{j} \in\left\{1, r_{i_{j}}\right\}$. La formule de localisation de Berline-Vergne va donc exprimer notre intégrale en termes d'une somme indexée par ces $\ell$-uplets :

$$
\int_{\Gamma_{w}} \mu(Y)=(-\sqrt{-1})^{\ell} \sum_{\left(\sigma_{1}, \ldots, \sigma_{\ell}\right)} \frac{\Theta(\mu)\left(\sigma_{1} \cdots \sigma_{\ell}\right)(Y)}{\operatorname{Pf}\left(L(Y)\left(\sigma_{1}, \ldots, \sigma_{\ell}\right)\right)},
$$

(cf. [7][1]) où $L(Y)\left(\sigma_{1}, \ldots, \sigma_{\ell}\right)$ dénote la dérivée de Lie de l'action infinitésimale de $Y \in \mathfrak{t}$ sur l'espace tangent à $\Gamma_{w}$ au point fixe $\left(\sigma_{1}, \ldots, \sigma_{\ell}\right)$. Après calcul ( $c f .[1])$, on obtient

$$
\operatorname{Pf}\left(L(Y)\left(\sigma_{1}, \ldots, \sigma_{\ell}\right)\right)=(\sqrt{-1})^{\ell} \prod_{j=1}^{\ell}\left(\sigma_{1} \cdots \sigma_{j}\right)\left(\alpha_{i_{j}}\right)(Y),
$$

ce qui, remplacé dans (a), donne l'égalité annoncée par la proposition.

Remarque. - La fraction rationnelle $q_{w}^{w}$ vaut $\prod_{\gamma \in \Delta_{+} \cap w \Delta_{-}}\left(\gamma^{-1}\right)$.

Erratum. - Dans la note [3], le signe moins (-) devant le terme $\sigma_{1} \cdots \sigma_{j}\left(\alpha_{i_{j}}\right)$ de la formule définissant les $q_{w}^{u}$ (notés alors $q_{w^{\prime}}^{w}$ ) a été omis.

Notons, pour chaque élément $u \in W, \delta(u)$ la forme $Q$-linéaire sur $F(W ; Q)$ définie par $\delta(u)(f)=f(u)$. L'argument de la proposition précédente reste valable sur $\lim _{\overparen{W}}\left(H_{T}^{*}\left(\mathcal{F}_{m}\right) \otimes_{S} q\right)_{m>2 \lg (w)}$, et, après localisation, on a, pour tout $w \in \overleftarrow{W}$,

$$
L_{w}=\sum_{u \leq w} q_{w}^{u} \delta(u)
$$


3.4. Structure de $W$-module sur $H_{T}^{*}(\mathcal{F})$. - Soit $w$ un élément du groupe de Weyl $W$, la formule $w \cdot[p, m]=\left[p w^{-1}, w m\right]$ définit une action de $W$ sur $\mathbb{E} T \times{ }^{T} \mathcal{F}$ (resp. sur $\mathbb{E} T \times{ }^{T} \mathcal{F}^{i}$ et $\mathbb{B} T \times W$ ) induisant une structure de $W$-module sur $H_{T}^{*}(\mathcal{F})$ (resp. $H_{T}^{*}\left(\mathcal{F}^{i}\right)$ et $F_{b}\left(W ; S^{*}\right)$ ). L'interprétation de cette action sur $F_{b}\left(W ; S^{*}\right)$ (et même sur $F(W ; Q)$ ) est donnée par

$$
(w \cdot f)(u)=w\left(f\left(w^{-1} u\right)\right)
$$

pour tous $w$ et $u$ dans $W$ et $f$ dans $F_{b}\left(W ; S^{*}\right)$. La fibration $\mathbb{E} T \times{ }^{T} \mathcal{F} \rightarrow \mathbb{B} T$ (resp. $\mathbb{E} T \times{ }^{T} \mathcal{F}^{i} \rightarrow \mathbb{B} T$ et $\mathbb{B} T \times W \rightarrow \mathbb{B} T$ ) étant ainsi $W$-équivariante, les applications $\Theta: H_{T}^{*}(\mathcal{F}) \rightarrow H_{T}^{*}(W)$ et $\tilde{\pi}_{i}^{*}: H_{T}^{*}\left(\mathcal{F}^{i}\right) \rightarrow H_{T}^{*}(\mathcal{F})$ sont des homomorphismes de $W$-modules.

Lemme 3.4.1. - Soit $\mathcal{M}$ un sous-W-module de $F(W ; Q)$ (resp. $\left.H_{T}^{*}(\mathcal{F})\right)$. Tout endomorphisme $b$ de $W$-module de $\mathcal{M}$ est entièrement déterminé par la composée $L_{e} \circ b\left(\right.$ resp. $\left.\mathcal{L}_{e} \circ b\right)$, i.e. pour tout endomorphisme de $W$-module $b^{\prime}$ de $\mathcal{M}$, on aura $b=b^{\prime}$ si et seulement si, $L_{e} \circ b=L_{e} \circ b^{\prime}\left(\right.$ resp. $\left.\mathcal{L}_{e} \circ b=\mathcal{L}_{e} \circ b^{\prime}\right)$.

Démonstration. - Sur $F(W ; Q)$ ceci résulte de l'égalité évidente

$$
b(f)(w)=w\left(\left(L_{e} \circ b\right)\left(w^{-1} \cdot f\right)\right) .
$$

L'injectivité de $\Theta$ implique le résultat sur $H_{T}^{*}(\mathcal{F})$.

Proposition 3.4.1. - Soient $r_{i_{1}}, \ldots, r_{i_{\ell}}$ des réflexions simples et posons $w=r_{i_{1}} \cdots r_{i_{\ell}}$. L'opérateur $A_{i_{1}} \circ \cdots \circ A_{i_{\ell}}$ est un endomorphisme de $W$-module de $F(W ; Q)$ satisfaisant à l'une des conditions suivantes :

(1) si $\lg (w)=\ell$, l'application $L_{w}$ détermine entièrement l'opérateur $A_{i_{1}} \circ \cdots \circ A_{i_{\ell}}$ par l'égalité

$$
\left(A_{i_{1}} \circ \cdots \circ A_{i_{\ell}}\right)(f)(v)=v\left(L_{w}\left(v^{-1} \cdot f\right)\right),
$$

pour tous $f \in F(W ; Q)$ et $v \in W$;

(2) si $\lg (w)<\ell$, on $a A_{i_{1}} \circ \cdots \circ A_{i_{\ell}}=0$.

Démonstration. - L'explicitation des $A_{i}$ et celle de l'action de $W$ sur $F(W ; Q)$ permettent de vérifier par un calcul élémentaire que chaque $A_{i}$ est un homomorphisme de $W$-module, i.e.

$$
A_{i}(w \cdot f)(v)=\left(w \cdot A_{i}(f)\right)(v)
$$

pour tous $w, v \in W$ et $f \in F(W ; Q)$. L'affirmation (1) découle alors du lemme précédent et de l'égalité $L_{e} \circ A_{i_{1}} \circ \cdots \circ A_{i_{\ell}}=L_{w}$, obtenue par

TOME $117-1989-\mathrm{N}^{\circ} 2$ 
itération du Corollaire 3.3.1. Enfin (2) est une conséquence classique de (1) (cf. [1] ou [2]).

Notation. - Cette proposition implique le caractère intrinsèque des composées des $\mathcal{A}_{i}$. Si $w$ est un élément de $W$, on notera respectivement $\mathcal{A}_{w}$ et $A_{w}$ les endomorphismes $\mathcal{A}_{i_{1}} \circ \cdots \circ \mathcal{A}_{i_{\ell}}$ et $A_{i_{1}} \circ \cdots \circ A_{i_{\ell}}$ obtenus à partir d'une décomposition réduite $\left(r_{i_{1}}, \ldots, r_{i_{\ell}}\right)$ de $w$.

Dans le paragraphe 3.7 , on donnera une interprétation cohomologique des $\mathcal{A}_{w}$ qui expliquera autrement leur unicité.

\subsection{Caractérisation algébrique de l'image de $\Theta$.}

THÉORÈmE 3.5.1. - L'homomorphisme de restriction $\Theta: H_{T}^{*}(\mathcal{F}) \rightarrow$ $F_{b}(W ; S)$ établit un isomorphisme entre $H_{T}^{*}(\mathcal{F})$ et l'ensemble des applications $f$ de $F_{b}(W ; S)$ satisfaisant à l'une quelconque des deux conditions suivantes :

(1) $A_{w}(f)(e) \in S$, pour tout $w$ dans $W$, où e dénote l'élément neutre de $W$;

(2) $A_{w}(f) \in F_{b}(W ; S)$ pour tout $w$ dans $W$.

En particulier l'image de $\Theta$ est la plus grande partie de $F_{b}(W ; S)$ stable sous l'action des $A_{i}$.

Démonstration. - Le fait que l'image de $\Theta$ soit caractérisée par la condition (1) est une conséquence immédiate de la Proposition 2.6.1(3) et de la Proposition 3.4.1(1) appliquée à $v=e$. L'équivalence des conditions (1) et (2) découle aussi de la Proposition 3.4.1 par le fait que l'image de $\Theta$ est $W$-stable, car alors

$$
A_{w}(f)(u)=u\left(L_{w}\left(u^{-1} \cdot f\right)\right) \in S,
$$

pour tout $u \in W$.

3.6. $W$-invariants de $H_{T}^{*}(\mathcal{F})$. - Dans les paragraphes précédents nous avons prouvé que $\Theta$ et les $A_{w}$ sont des homomorphismes de $W$ modules, par conséquent $\Theta\left(H_{T}^{*}(\mathcal{F})^{W}\right)$ est contenue dans la sous-algèbre $F_{b}(W ; S)^{W}$ et $F(W ; Q)^{W}$ est stable sous l'action des $A_{w}$.

Considérons l'application $L_{e}: F(W ; Q) \rightarrow Q$, d'évaluation en l'élément neutre $e$ de $\mathrm{W}$, i.e. $L_{e}(f)=f(e)$ pour tout $f \in F(W ; Q)$. La condition de $W$-invariance pour $f$ montre que $w(f(e))=f(w)$, on comprend donc que $L_{e}$ établisse un isomorphisme d'algèbres entre $F(W ; Q)^{W}$ et $Q$ et projette les $A_{w} \operatorname{sur} Q$.

Pour chaque $i=1,2, \ldots, n$, notons $\underline{A}_{i}$ l'opérateur sur $Q$ défini par

$$
\underline{A}_{i}(P)=\left(r_{i} P-P\right) \alpha_{i}^{-1}
$$


pour tout $P$ dans $Q$. On vérifie aisément l'égalité $\underline{A}_{i} \circ L_{e}=L_{e} \circ A_{i}$ et le fait que $\underline{A}_{i}$ stabilise $S$. Ces conditions entraînent la stabilité de $F(W ; S)^{W}$ sous l'action des $A_{i}$ et l'inclusion $F(W ; S)^{W} \subseteq \operatorname{Im}(\Theta)$ découle alors en appliquant le THÉORÈme 3.5.1. La proposition suivante se trouve ainsi prouvée.

Proposition. - L'application $\mathcal{L}_{e}=L_{e} \circ \Theta$ établit un isomorphisme d'algèbres gaduées entre $H_{T}^{*}(\mathcal{F})^{W}$ et $S^{*}$.

3.7. Interprétation cohomologique des endomorphismes $\mathcal{A}_{w}$. Faisons agir $G$ sur le produit cartésien $\mathcal{F} \times \mathcal{F}$ par $g(x, y)=(g x, g y)$ pour tous $x, y \in \mathcal{F}$ et $g \in G$. Notons $p_{1}$ et $p_{2}$ les projections de $\mathcal{F} \times \mathcal{F}$ sur $\mathcal{F}$ définies par $p_{1}(x, y)=x$ et $p_{2}(x, y)=y$ respectivement. Pour chaque élément $w \in W$ notons $\mathcal{T}_{w}$ la partie de $\mathcal{F} \times \mathcal{F}$ obtenue en saturant le produit de variétés de Schubert $\overline{\mathcal{F}}_{e} \times \overline{\mathcal{F}}_{w}$ par l'action de $G$. Par restriction de $p_{1}$ à $\mathcal{T}_{w}$, on obtient une fibration $G$-équivariante canoniquement orientée, de fibre $\overline{\mathcal{F}}_{w}$ et de base $\mathcal{F}$. (Ces fibrations m'ont été indiquées par M. Demazure.) Restreignons $p_{2}$ à $\mathcal{T}_{w}$ et posons $\mathcal{D}_{w}=\tilde{p}_{1 *} \circ \tilde{p}_{2}^{*}$; c'est un endomorphisme de $H_{T}^{*}(\cdot)$-module de $H_{T}^{*}(\mathcal{F})$.

Proposition 3.7.1. - Quel que soit l'élément $w$ de $W$, il y a égalité entre $\mathcal{A}_{w}$ et $\mathcal{D}_{w}$.

Nous allons donner deux démonstrations, la première utilisera certains des résultats des paragraphes précédents alors que la seconde en sera indépendante.

Démonstration I. - Remarquons tout d'abord l'égalité $\mathcal{L}_{e} \circ \mathcal{D}_{w}=$ $\mathcal{L}_{e} \circ \mathcal{A}_{w}$. Pour ceci, considérons le diagramme commutatif $T$-équivariant

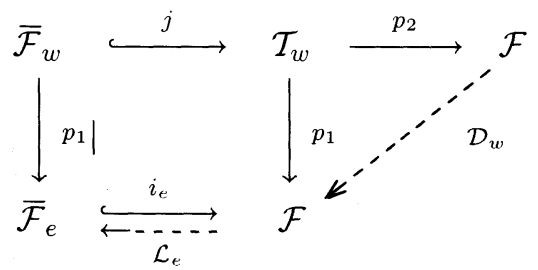

où $j$ dénote l'application $j(x)=(e, x)$ et où $i_{e}$ dénote l'injection canonique $\operatorname{de} \overline{\mathcal{F}}_{e}$ dans $\mathcal{F}$. (Par abus de notation, $e$ dénote à la fois l'élément neutre de $G$ et la classe qu'il détermine dans $\mathcal{F}=G / B$ ) et $p_{1} \mid$ dénote la restriction de $p_{1}$ à $\overline{\mathcal{F}}_{w}$.) La naturalité de l'intégration sur les fibres nous fournit l'égalité

$$
\mathcal{L}_{e} \circ \mathcal{D}_{w}=\tilde{\imath}_{e}^{*} \circ \tilde{p}_{1 *} \circ \tilde{p}_{2}^{*}=\left.\tilde{p}_{1}\right|_{*} \circ \tilde{\jmath}^{*} \circ \tilde{p}_{2}^{*}=\mathcal{L}_{w}
$$

TOME $117-1989-\mathrm{N}^{\circ} 2$ 
car $p_{2} \circ j$ est l'injection canonique de $\overline{\mathcal{F}}_{w}$ dans $\mathcal{F}$. Ceci étant, le Lemme 3.4.1 permet de conclure à l'égalité entre $\mathcal{A}_{w}$ et $\mathcal{D}_{w}$ puisque $\mathcal{D}_{w}$ est un homomorphisme de $W$-modules, ce qui résulte de l'observation que, dans l'égalité $\mathcal{D}_{w}=\tilde{p}_{1 *} \circ \tilde{p}_{2}^{*}$, l'homomorphisme $\tilde{p}_{2}^{*}$ respecte bien les structures de $W$ modules de même que $\tilde{p}_{1 *}$ comme le montre un argument de topologie algébrique élémentaire.

L'idée de la démonstration suivante est dûe à $M$. Demazure.

Démonstration II. - Commençons par remarquer que si $r_{i}$ est une réflexion simple, on a bien $\mathcal{D}_{r_{i}}=\mathcal{A}_{i}$. Par définition de $\mathcal{A}_{i}$ et par la naturalité de l'intégration sur les fibres, nous avons, d'après le diagramme $T$-équivariant commutatif

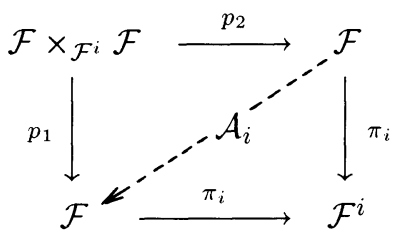

l'égalité $\mathcal{A}_{i}=\tilde{\pi}_{i}^{*} \circ \tilde{\pi}_{i *}=\tilde{p}_{1 *} \circ \tilde{p}_{2}^{*}$. Or il est tout à fait immédiat que $\mathcal{F} \times_{\mathcal{F}^{i}} \mathcal{F}$ coïncide avec $\mathcal{T}_{r_{i}}$, ce qui prouve notre affirmation. Ceci étant, nous montrerons dans la suite que si $r_{i}$ est une réflexion simple et $w$ est un élément de $W$ vérifiant $w<r_{i} w$, on a l'égalité $\mathcal{D}_{r_{i} w}=\mathcal{D}_{r_{i}} \circ \mathcal{D}_{w}$. Par itération on obtiendra $\mathcal{D}_{w}=\mathcal{A}_{i_{1}} \circ \cdots \circ \mathcal{A}_{i_{\ell}}$ pour toute décomposition réduite $\left(r_{i_{1}}, \ldots, r_{i_{\ell}}\right)$ de $w$, ce qui terminera la démonstration. Supposons donc $r_{i} w>w$ et considérons le diagramme $T$-équivariant $\left(\mathrm{D}^{\prime}\right)$ de la page suivante, où $q_{1}$ et $q_{2}$ sont les projections habituelles du produit fibré de $\pi_{i}$ et $\pi_{i} \circ p_{1}$ respectivement. Nous avons alors

$$
\mathcal{D}_{r_{i}} \circ \mathcal{D}_{w}=\tilde{\pi}_{i}^{*} \circ \tilde{\pi}_{i *} \circ \tilde{p}_{1 *} \circ \tilde{p}_{2}^{*}=\tilde{q}_{1 *} \circ\left(\tilde{p}_{2} \circ \tilde{q}_{2}\right)^{*}
$$

Précisons la nature de la fibration $q_{1}$. Sa fibre $F$ est, par la définition même du produit fibré, donnée comme l'ensemble des couples d'éléments de $\mathcal{F}$ de la forme $(g \cdot e, g x \cdot e)$ où $g \in P^{i}$ et $x \in \bar{G}_{w}=\bigcup_{w^{\prime} \leq w} B w^{\prime} B$ et où $e$ dénote la clase de l'élément neutre de $G$ dans $\mathcal{F}$. D'autre part l'espace $\mathcal{F} \times{ }_{\mathcal{F}^{i}} \mathcal{T}_{w}$ est constitué des triplets d'éléments de $\mathcal{F}$ de la forme $(g \cdot e, g y \cdot e, g y x \cdot e)$ où $g \in G, y \in P^{i}$ et $x \in \bar{G}_{w}$. L'égalité $P^{i} \bar{G}_{w}=\bar{G}_{r_{i} w}$ permet alors de définir un morphisme $s: \mathcal{F} \times_{\mathcal{F}^{i}} \mathcal{T}_{w} \rightarrow \mathcal{T}_{r_{i} w}$ de fibrations au-dessus de $\mathcal{F}$ par $s(a, b, c)=(a, c)$, ce qui fournit le diagramme 


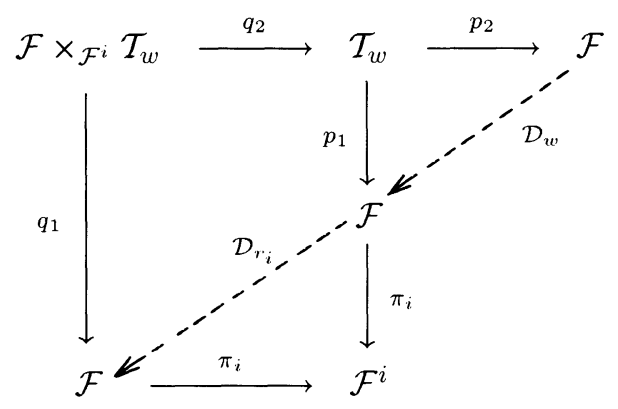

Diagramme $\left(\mathrm{D}^{\prime}\right)$

commutatif et $T$-équivariant

$p_{2} \circ q_{2}$

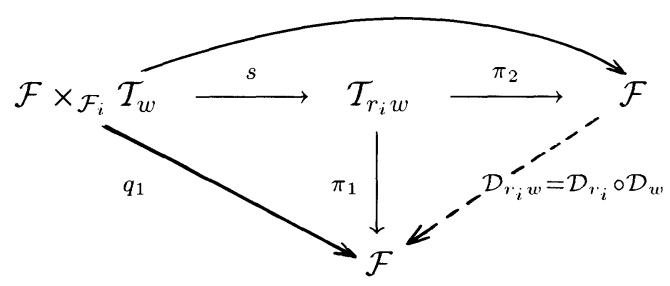

Il résute de ce diagramme l'égalité

$$
\tilde{q}_{1 *} \circ\left(\tilde{p}_{2} \circ \tilde{q}_{2}\right)^{*}=\tilde{q}_{1 *} \circ \tilde{s}^{*} \circ \tilde{\pi}_{2}^{*}=\tilde{\pi}_{1 *} \circ \tilde{\pi}_{2}^{*}=\mathcal{D}_{r_{i} w},
$$

qui, avec (1), achève la démonstration de la proposition.

Remarque. - Dans le cas où $w>r_{i} w$, ces idées permettent de prouver l'égalité $\mathcal{D}_{r_{i}} \circ \mathcal{D}_{w}=0$. En effet, on aura, contrairement au cas précédent, $P^{i} \bar{G}_{w}=\bar{G}_{w}$ et le morphisme $s$ sera donc à valeurs dans $\mathcal{T}_{w}$. Ainsi, dans le diagramme $(\mathcal{D})$ précédent, la fibre de $q_{1}$ sera de dimension $2(\lg (w)+1)$, strictement plus grande que celle de $\pi_{1}$, impliquant l'égalité $\tilde{q}_{1 *} \circ \tilde{s}^{*}=0$, d'où l'affirmation.

Ces raisonnements prouvent directement que pour tous $w_{1}$ et $w_{2}$ dans $W$, on a ( $c f$. Proposition 3.4.1) les égalités suivantes :

TOME $117-1989-\mathrm{N}^{\mathrm{O}} 2$ 
(1) $\mathcal{D}_{w_{1}} \circ \mathcal{D}_{w_{2}}=\mathcal{D}_{w_{1} w_{2}}$ si $\lg \left(w_{1} w_{2}\right)=\lg \left(w_{1}\right)+\lg \left(w_{2}\right)$;

(2) $\mathcal{D}_{w_{1}} \circ \mathcal{D}_{w_{2}}=0$ autrement.

\section{Liaisons avec les résultats annoncés par B. Kostant et S. Kumar}

Dans cette section nous indiquons comment les développements précédents expliquent les principaux résultats de la note [12] de B. Kostant et S. Kumar. Celle-ci se trouve d'ailleurs à l'origine de notre étude.

4.1. Algèbre $Q_{W}$ d'opérateurs sur $F(W ; Q)$. - Soit $\mathbb{Z}[W]$ la $\mathbb{Z}$-algèbre de $W$, c'est-à-dire le $\mathbb{Z}$-module libre engendré par la famille $\left\{\delta_{w}\right\}_{w \in W}$ et dont le produit découle des égalités $\delta_{w} \delta_{v}=\delta_{w v}$. Posons $Q_{W}=\mathbb{Z}[W] \otimes Q$ et munissons-le de la structure d'algèbre donnée par le produit

$$
\left(\delta_{w} \otimes Q_{1}\right) \cdot\left(\delta_{v} \otimes Q_{2}\right)=\delta_{w v} \otimes\left(v^{-1} Q_{1}\right) Q_{2} .
$$

Considérons $Q_{W}$ munie de la structure naturelle de $Q$-module $\grave{a}$ droite et posons suivant Kostant et $\operatorname{Kumar} \Omega=\operatorname{Hom}_{Q}\left(Q_{W}, Q\right)$, module des homomorphismes de $Q$-modules à droite. Les auteurs considèrent ensuite l'anti-automorphisme involutif $a \mapsto a^{t}$ sur $Q_{W}$ défini par $\left(\delta_{w} \otimes q\right)^{t}=$ $\delta_{w^{-1}} \otimes w(q)$ et font opérer $Q_{W}$ sur $\Omega$ suivant la formule

$$
(a \cdot \psi)(b)=\psi\left(a^{t} \cdot b\right),
$$

pour tous $a, b$ dans $Q_{W}$ et $\psi$ dans $\Omega(c f$. [12]).

Considérons l'isomorphisme de $Q$-modules à droite $\left(^{\wedge}\right)$ entre $F(W ; Q)$ et $\Omega$ défini par

$$
\hat{f}\left(\delta_{w} \otimes 1\right)=f\left(w^{-1}\right),
$$

pour tout $f$ appartenant à $F(W ; Q)$. Un calcul élémentaire prouve le :

Lemme. - L'action de l'endomorphisme $A_{i}$ sur $F(W ; Q)$ se projette sur $\Omega$, modulo l'isomorphisme (^), par l'action de l'élément

$$
x_{i}=\left(-\delta_{r_{i}}-\delta_{e}\right) \otimes \alpha_{i}^{-1}
$$

appartenant $\grave{a} Q_{W}$.

Résultat qui explique l'assertion suivante.

Proposition 4.1.1 [12]. - Soient $r_{i_{1}}, \ldots, r_{i_{\ell}}$ des réflexions simples de $W$, l'élément $x_{i_{1}} \cdots x_{i_{\ell}}$ de $Q_{W}$ satisfait aux conditions suivantes :

(1) si $\lg \left(r_{i_{1}} \cdots r_{i_{\ell}}\right)=\ell$, l'élément $x_{i_{1}} \cdots x_{i_{\ell}}$ seul dépend du produit $w=r_{i_{1}} \cdots r_{i_{\ell}}$ et non pas des compositions réduites considérées; 
(2) $s i \lg \left(r_{i_{1}} \cdots r_{i_{\ell}}\right)<\ell$, on a $x_{i_{1}} \cdots x_{i_{\ell}}=0$.

Démonstration. - C'est la Proposition 3.4.1, moyennant le fait que l'action de $Q_{W}$ sur $\Omega$ est fidèle.

Notation. - Pour chaque élément $w \in W$, on notera $x_{w}$ l'unique élément de $Q_{W}$ réalisant l'action de $A_{w}$.

Remarque. - L'ensemble $\left\{x_{w}\right\}_{w \in W}$ détermine une base de $Q_{W}$. Une question d'intérêt considérée par KoSTANT et Kumar dans leur article est la détermination de la matrice de changement de base de $\left\{\delta_{w} \otimes 1\right\}$ à $\left\{x_{w}\right\}$ pour la structure de $Q$-module à gauche sur $Q_{W}$. Dans [12,13] ils posent

$$
x_{w}=\sum_{v \leq w} c_{w^{-1}}^{v^{-1}}\left(\delta_{v} \otimes 1\right)
$$

pour tout $w \in W$. Au sujet des coefficients $c_{w}^{u}$, je voudrais signaler l'égalité $c_{w^{-1}}^{u^{-1}}=q_{w}^{u}(c f$. Proposition 3.3.1) et renvoie le lecteur désireux de plus de renseignements aux articles en question. En effet, on a les égalités :

(1) $L_{w}=L_{e} \circ A_{w}=L_{e} \circ x_{w}$;

(2) $\delta(u)=L_{e} \circ\left(\delta_{u} \otimes 1\right)$;

d'où l'identité avancée moyennant l'égalité ( $\ddagger)$ du paragraphe 3.3 puisque le système $\{\delta(u)\}_{u \in W}$ est libre.

4.2. Sous-module $\Lambda$ et cohomologie de $\mathcal{F}$. - Un objet important de [12] et [13] est la partie $\Lambda$ de $\Omega$ définie comme l'ensemble des homomorphismes $\hat{f}$ satisfaisant aux deux conditions suivantes :

(a) $\hat{f}\left(x_{w}^{t}\right) \in S$ pour tout $w \in W$;

(b) $\hat{f}\left(x_{w}^{t}\right)=0$ pour presque tout $w \in W$.

En munissant $\Omega$ de la structure d'algèbre transportée par $\left(^{\wedge}\right)$ de $F(W ; Q)$, Kostant et Kumar prouvent les résultats rassemblés dans le théorème suivant.

THÉORÈme 4.2.1. [12, 13].

(1) $\Lambda$ est une $S$-sous-algèbre de $\Omega$;

(2) $\Lambda$ est un $S$-module libre admettant une base $\left\{\xi^{w}\right\}_{w \in W}$ canoniquement caractérisée, pour tous $v, w \in W$, par

$$
\xi^{w}\left(x_{v}^{t}\right)=\delta_{v}^{w}
$$

(3) $\Lambda$ est stable sous l'action des $x_{w} \in Q_{W}$;

TOME $117-1989-\mathrm{N}^{\circ} 2$ 
(4) posons $Z_{0}=S / S^{+}$. L'application $\theta: \Lambda \otimes_{S} Z_{0} \rightarrow H^{*}(\mathcal{F})$ définie $\operatorname{par} \theta\left(\xi^{w} \otimes 1\right)=\mu^{w}$, où $\mu^{w}$ est la classe de cohomologie duale de la cellule $\overline{\mathcal{F}}_{w} \subseteq \mathcal{F}$, est un isomorphisme d'algèbres graduées.

Dans (4), $S^{+}$dénote l'idéal d'augmentation de $S^{*}$ et la graduation de $\Lambda$ est celle qui découle de l'équivalence $\Lambda \equiv H^{*}(\mathcal{F}) \otimes S^{*}$ annoncée dans (2).

Démonstration. - Soit $\hat{f}$ un élément de $\Lambda$. Par définition, nous savons que $\hat{f}\left(x_{w}^{t}\right)=L_{w}(f)$ appartient à $S$ et est presque toujours nul relativement aux $w \in W$, il existe donc un entier positif $N$ tel que $\sin \lg (w)>N$, on a $L_{w}(f)=0$. Montrons que $f$ est nécessairement de degré borné. Pour ceci, le plus rapide est d'utiliser l'explicitation des $L_{w}$ (sous une forme moins précise que l'on peut obtenir facilement par induction) de la Proposition 3.3.1. Ceci permettra de prouver par récurrence $\operatorname{sur} \lg (u)$ que le degré de $f(u)$ est majoré par le plus grand des degrés de la famille finie $\{f(v)\}_{\lg (v) \leq N}$. En effet nous avons $L_{u}(f)=\sum_{v \leq u} q_{u}^{v} f(v)$ et donc, si $\lg (u)>N$, on a

$$
q_{u}^{u} f(u)=-\sum_{v<u} q_{u}^{v} f(v)
$$

d'où la conclusion par hypothèse de récurrence puisque les $q_{u}^{v}$ sont homogènes de degré $-\lg (u)$ et que $q_{u}^{u} \neq 0$ ( $c f$. Remarque paragraphe 3.3).

Ainsi moyennant l'isomorphisme $\left(^{\wedge}\right)$, la partie $\Lambda$ est contenue dans $F_{b}(W ; S)$ où l'on reconnaît dans la condition $(a)$ de sa définition la caractérisation de l'image de l'homomorphisme $\Theta$ qui vient nous identifier $\Lambda$ à $H_{T}^{*}(\mathcal{F})$. On remarquera que les éléments $\xi^{w}$ correspondent aux $\tilde{\mu}^{w}$ que nous avons définis dans le paragraphe 2.5. Enfin (3) est clair et (4) découle de la Proposition 2.4.1 (3) qui donne le diagramme commutatif

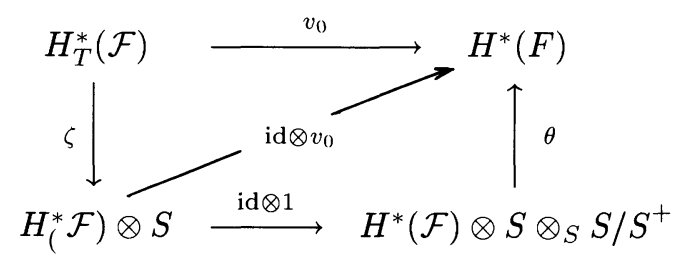

où $\zeta$ est l'isomorphisme canonique de la Proposition 2.5.1 et bien qu'il ne respecte pas la structure d'algèbre, suffit pour prouver la bijectivité de $\theta$ qui est, par ailleurs, trivialement un homomorphisme d'algèbres graduées. 


\section{Appendice}

Le but de cette dernière section est de décrire le procédé d'intégration sur les fibres dans le contexte des fibrations de Serre orientables où la fibre est munie d'une structure de $\mathrm{CW}$-complexe finie. Je tiens à signaler qu'il ne sera nullement supposé de condition de finitude de dimension sur la base.

5.1. Image directe relative à une classe de la fibre. - Soit $M$ l'espace total d'une fibration orientable ( $c f$. [14]) de base $N$ et de fibre $F$, notons $\pi: M \rightarrow N$ la projection correspondante. Supposons $F$ munie d'une structure de $\mathrm{CW}$-complexe et soit $\sigma$ une classe d'homologie de $F$ de dimension $d$, où

(1) $H_{d}(F)$ est un $\mathbb{Z}$-module libre;

(2) $H_{d+j}(F) \equiv 0$ pour tout $j \geq 1$.

Ces données constituent ce que nous désignerons dans la suite par fibration orientée (de classe $\sigma$ ).

Le module bigradué $E_{2}^{*, \bullet}$ de la suite spectrale de Leray-Serre de cette fibration est isomorphe, en tant qu'algèbre graduée, à $H^{*}\left(N ; H^{\bullet}(F)\right)$. Par conséquent, son terme $E_{2}^{p, q}$ sera nul, grâce aux conditions (1) et (2) cidessus, lorsque $q>d$. On en déduit, d'une part, que pour $q>d$ on a $E_{r}^{*, q}=E_{2}^{*, q}=0$ pour tout $r \geq 2$, et, d'autre part, que pour $r \geq 2$ la différentielle $d_{r}: E_{r}^{*-r, d+r-1} \rightarrow E_{r}^{*, d}$ est nulle. Ces remarques entraînent l'inclusion $E_{\infty}^{*, d} \subseteq E_{2}^{*, d}$ et nous avons donc les égalités

$$
H^{p+d}(M)=H^{p+d}(M)_{1}=\cdots=H^{p+d}(M)_{p},
$$

pour tout entier $p$, ainsi qu'un homomorphisme de $H^{*}(N)$-modules composé de

$$
H^{p+d}(M) \stackrel{\alpha}{\longrightarrow} H^{p+d}(M)_{p} / H^{p+d}(M)_{p+1} \longleftrightarrow E_{2}^{p, d},
$$

où $\alpha$ dénote la projection canonique.

Enfin, on a l'homomorphisme de $H^{*}(N)$-modules noté $I_{\sigma}$ de $E_{2}^{*, d} \equiv$ $H^{*}\left(N ; H^{d}(F)\right)$ à valeurs dans $H^{*}(N)$ induit par $\sigma$, d'où l'application composée :

$$
H^{p+d}(M) \stackrel{\alpha}{\longrightarrow} H^{p+d}(M)_{p} / H^{p+d}(M)_{p+1} \stackrel{I_{\sigma}}{\longrightarrow} H^{p}(N) .
$$

Définition. - Soit $\pi: M \rightarrow N$ une fibration de fibre $F$ orientée, de classe d'homologie $\sigma$ de dimension $d$. On appelle image directe relative $\grave{a} \sigma$ et on note $\pi_{\sigma}$, l'homomorphisme de $H^{*}(N)$-modules gradués $\pi_{\sigma}$ :

TOME $117-1989-\mathrm{N}^{\circ} 2$ 
$H^{*}(M) \rightarrow H^{*-d}(N)$, nul sur $H^{p}(M)$ pour $p<d$ et défini par ( $\dagger$ ) pour tout $p \geq d$.

Remarques. - Lorsque $N$ est réduit à un point, on retrouve la définition du cap-produit sur $M$ habituel. En utilisant la suite spectrale de Leray-Serre relative, on définit pareillement $\pi_{\sigma}: H^{*}\left(M, M^{\prime}\right) \rightarrow$ $H^{*-d}\left(N, N^{\prime}\right)$, où $M^{\prime}=\pi^{-1}\left(N^{\prime}\right)$.

Proposition 5.1.1. - Soit $(\mu, \nu)$ un morphisme de fibrations de la fibration orientée $\pi_{1}$ de fibre $F_{1}$ de classe $\sigma$ de dimension $d_{1}$, dans la fibration orientable $\pi_{2}$ de fibre $F_{2}$. Supposons en plus que:

(1) $H_{d_{1}}\left(F_{2}\right)$ est un $\mathbb{Z}$-module libre;

(2) $H_{d_{1}+j}\left(F_{2}\right) \equiv 0$ pour tout $j \geq 1$.

Notons $\mu_{*}$ le morphisme de $H_{*}\left(F_{1}\right)$ à valeurs dans $H_{*}\left(F_{2}\right)$ induit par $\mu$. Alors

$$
\pi_{1, \sigma} \circ \mu^{*}=\nu^{*} \circ \pi_{2, \mu_{*}(\sigma)}
$$

En particulier si $F_{2}$ est de dimension finie $d_{2}$ strictement majorée par $d_{1}$, on $a \pi_{1, \sigma} \circ \mu^{*}=0$.

Démonstration. - Notons $\left(\pi_{1}, M_{1}, N_{1}, F_{1}\right)$ et $\left(\pi_{2}, M_{2}, N_{2}, F_{2}\right)$ les fibrations. Les conditions (1) et (2) donnent un sens à l'application $\alpha_{2} \mathrm{du}$ diagramme suivant.

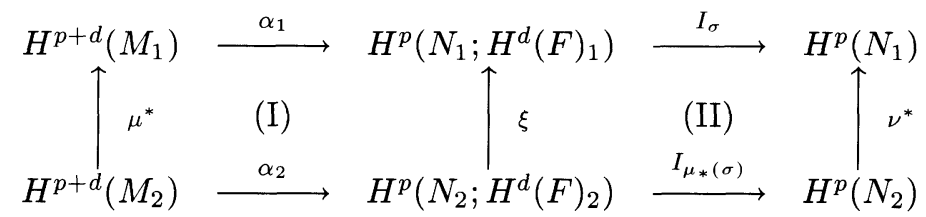

La naturalité de la suite spectrale de Leray-Serre garantit la commutativité du sous-diagramme (I). Enfin (II) commute aussi puisque $\xi$ n'est autre que l'application naturelle induite par les morphismes d'image réciproque $\nu^{*}$ et $\mu^{*}: H^{d}\left(F_{2}\right) \rightarrow H^{d}\left(F_{1}\right)(c f .[14])$

Corollaire 5.1.1. - Soit $\pi: M \rightarrow N$ une fibration orientée, de fibre de classe $\sigma$ de dimension $d>0$. Alors :

(1) $\pi_{\sigma} \circ \pi^{*}=0$;

(2) l'application $\pi^{*}$ o $\pi_{\sigma}$ est un endomorphisme du $H^{*}(N)$-module gradué $H^{*}(M)$, de degré -d et de carré nul.

Démonstration. - Il suffit d'appliquer la proposition précédente au morphisme de fibrations $\left(\pi, \operatorname{id}_{N}\right)$ de $(\pi, M, N, F)$ dans $\left(\operatorname{id}_{N}, N, N, \cdot\right)$.

Remarque. - Lorsque la structure de CW-complexe de la fibre $F$ est de dimension finie $d$ et ne comporte qu'un nombre fini de cellules de 
dimension maximum, leurs paramétrisations déterminent des $d$-simplexes singuliers dont la somme $\Sigma$ est une $d$-chaîne singulière canonique. On dira que $F$ est un $C W$-complexe orienté si le bord de $\Sigma$ est de codimension deux modulo un bord de codimension un, i.e.

$$
\partial \Sigma \in B_{d-1}\left(F_{d-1}\right)+Z_{d-1}\left(F_{d-2}\right),
$$

où $F_{k}$ dénote le $k$-squelette de $F$. La chaîne $\Sigma$ définit, dans ce cas, un $d$-cycle non trivial du complexe sphérique associé à $M$ et donc une classe d'homologie $\sigma$ de degré $d$ qu'on appellera la classe fondamentale $d u C W$ complexe orienté $F$. Toute fibration orientable $\pi$ de fibre $F$ sera alors canoniquement orientée et le morphisme d'image directe $\pi_{\sigma}$ sera appelé intégration sur les fibres et noté simplement par $\pi_{*}$ et même $\int_{F}$ lorsque la base de la fibration sera réduite à un point.

Les variétés de Schubert de $G / B$ et de $G / P^{i}$ admettent une structure canonique de $\mathrm{CW}$-complexes ne possédant pas de cellule de dimension impaire; celle-ci les rend donc automatiquement orientées. On remarquera que dans ce cas, l'orientation obtenue est précisément celle associée à la structure complexe des cellules de Bruhat.

Dans la section suivante on précisera le comportement de l'intégration sur les fibres lors des compositions de fibrations.

\subsection{Factorisation de l'intégration sur les fibres.}

LEMme 5.2.1. - Soit $\pi: F \rightarrow F_{2}$ une fibration orientable de fibre $F_{1}$. Supposons les espaces $F, F_{1}$ et $F_{2}$ munis de structures de $C W$ complexes finies de dimensions $d, d_{1}$ et $d_{2}$ respectivement. Il existe alors deux isomorphismes canoniques :

(a) $H_{d}(F) \equiv H_{d_{2}}\left(F_{2}\right) \otimes H_{d_{1}}\left(F_{1}\right)$;

(b) $H^{d}(F) \equiv H^{d_{2}}\left(F_{2} ; H^{d_{1}}\left(F_{1}\right)\right)$.

Démonstration. - Construisons, dans un premier temps, l'isomorphisme (b). Dans la suite spectrale pour la fibration $\pi$ le terme $E_{2}^{p, q} \equiv H^{p}\left(F_{2} ; H^{q}\left(F_{1}\right)\right)$ est nul si $q>d_{1}$ ou si $p>d_{2}$ puisque, dans la suite exacte courte

$$
\begin{aligned}
0 \longrightarrow \operatorname{Ext}^{1}\left(H_{p-1}\left(F_{2}\right) ; H^{q}\left(F_{1}\right)\right) \longrightarrow & H^{p}\left(F_{2} ; H^{q}\left(F_{1}\right)\right) \rightarrow \\
& \rightarrow \operatorname{Hom}\left(H_{p}\left(F_{2}\right) ; H^{q}\left(F_{1}\right)\right) \longrightarrow 0
\end{aligned}
$$

$H_{p-1}\left(F_{2}\right)$ est libre et $H_{p}\left(F_{2}\right)$ est nul si $p>d_{2}$. Par conséquent

$$
E_{2}^{p, q}=0 \text { pour tout } 2 \leq r \leq \infty \text { et tout } p>d_{2} \text { ou } q>d_{1}
$$

TOME $117-1989-\mathrm{N}^{\circ} 2$ 
Ainsi les différentielles des suites

$$
\longrightarrow E_{r}^{d_{2}-r, d_{1}+r-1} \stackrel{d_{r}}{\longrightarrow} E_{r}^{d_{2}, d_{1}} \stackrel{d_{r}}{\longrightarrow} E_{r}^{d_{2}+r, d_{1}-r+1} \longrightarrow
$$

sont toutes nulles pour $r \geq 2$ d'où il résulte $E_{\infty}^{d_{2}, d_{1}} \equiv H^{d_{2}}\left(F_{2} ; H^{d_{1}}\left(F_{1}\right)\right)$. Or les quotients successifs du module filtré $H^{m}(F)_{*}$ sont les $E_{\infty}^{p, m-p}$ et, d'après ( $\dagger$ ), seront tous nuls si $m>d_{1}+d_{2}$. Mais, lorsque $m=d_{1}+d_{2}$, il n'y a qu'un seul terme éventuellement non nul, savoir $E_{\infty}^{d_{2}, d_{1}}$. On conclut, d'une part, que $d=d_{1}+d_{2}$ et, d'autre part, que $H^{d}(F) \equiv E_{\infty}^{d_{2}, d_{1}}$, ce qui prouve notre assertion.

Par un argument parfaitement dual, on obtient un isomorphisme $H_{d}(F) \equiv H_{d_{2}}\left(F_{2} ; H_{d_{1}}\left(F_{1}\right)\right)$. En considérant la suite exacte courte

$$
\begin{aligned}
0 \longrightarrow H_{d_{2}}\left(F_{2}\right) \otimes H_{d_{1}}\left(F_{1}\right) & \longrightarrow H_{d_{2}}\left(F_{2} ; H_{d_{1}}\left(F_{1}\right)\right) \rightarrow \\
& \rightarrow \operatorname{Tor}_{1}\left(H_{d_{2}-1}\left(F_{2}\right) ; H_{d_{1}}\left(F_{1}\right)\right) \longrightarrow 0
\end{aligned}
$$

il résulte un isomorphisme $H_{d_{2}}\left(F_{2} ; H_{d_{1}}\left(F_{1}\right)\right) \equiv H_{d_{2}}\left(F_{2}\right) \otimes H_{d_{1}}\left(F_{1}\right)$ puisque $H_{d_{1}}\left(F_{1}\right)$ est sans torsion.

Corollaire 5.2.1. - Soient $\pi_{1}$ et $\pi_{2}$ deux fibrations orientables de fibres $F_{1}$ et $F_{2}$ et de dimensions $d_{1}$ et $d_{2}$ respectivement. Supposons en plus que la base de $\pi_{1}$ coïncide avec l'espace total de $\pi_{2}$. Notons $F$ la fibre de la fibration composée $\pi_{2} \circ \pi_{1}$ et $d$ sa dimension. On a alors un isomorphisme canonique $H_{d}(F) \equiv H_{d_{2}}\left(F_{2}\right) \otimes H_{d_{1}}\left(F_{1}\right)$.

Démonstration. - Il suffit de remarquer que la fibration induite $\pi_{1}: F \rightarrow F_{2}$ de fibre $F_{1}$ est orientable puisque l'action du groupe fondamental $\Pi_{1}\left(F_{2}\right)$ sur $H_{*}\left(F_{1}\right)$ se factorise par celle, triviale, de $\Pi_{1}\left(M_{1}\right)$ où $M_{1}$ dénote l'espace total de la fibration $\pi_{1}$. On applique alors le lemme précédent.

La proposition suivante joue un rôle important dans les démonstrations des Propositions 3.2.1 et 3.7.1.

Proposition 5.2.1. - Soit $\pi$ une fibration orientée de classe fondamentale $\sigma$. Considérons une factorisation $\pi=\pi_{2} \circ \pi_{1}$, où $\pi_{1}$ et $\pi_{2}$ sont des fibrations orientées de classes fondamentales $\sigma_{1}$ et $\sigma_{2}$ respectivement. Supposons que, moyennant l'isomorphisme du corollaire précédent, on ait $\sigma=\sigma_{2} \otimes \sigma_{1}$. Alors, on a la factorisation d'intégrations sur les fibres

$$
\pi_{*}=\pi_{2, *} \circ \pi_{1, *}
$$

Démonstration. - Commençons par fixer la notation à $M \stackrel{\pi_{1}}{\longrightarrow} M_{1} \stackrel{\pi_{2}}{\longrightarrow} N$ et soient $F, F_{1}, F_{2}$ les fibres de $\pi, \pi_{1}$ et $\pi_{2}$ respectivement. Posons 
$\varphi=\pi_{*}-\pi_{2, *} \circ \pi_{1, *}$ et notons $\varphi^{p}$ sa restriction à $H^{p}(M)$. Cette restriction est à valeurs dans $H^{p-d}(N)$, où $d$ dénote la dimension de $F$. Notons $d_{1}$ et $d_{2}$ les dimensions respectives de $F_{1}$ et $F_{2}$ et supposons, dans un premier temps, que $N$ est de dimension finie $m$. Des raisonnements élémentaires de dimension montrent déjà que $\varphi^{p}$ sera nulle pour tout $p<d$ et pour tout $p>d+m$. Un peu moins évident est le fait que $\varphi^{d+m}$ est nul. En appliquant le Lemme 5.2.1, on a, pour la fibration $\pi$, l'isomorphisme

$$
H^{d+m}(M) \equiv H^{m}\left(N ; H^{d}(F)\right),
$$

et, pour les fibrations $\pi_{1}$ et $\pi_{2}$, l'isomorphisme

$$
\begin{aligned}
H^{d_{1}+d_{2}+m}(M) & \equiv H^{d_{2}+m}\left(M_{1} ; H^{d_{1}}\left(F_{1}\right)\right) \\
& \equiv H^{m}\left(N ; H^{d_{2}}\left(F_{2} ; H^{d_{1}}\left(F_{1}\right)\right)\right) .
\end{aligned}
$$

L'action de $\pi_{*}$ se lit sur $(a)$ comme celle de $\sigma$ et celle de $\pi_{2, *} \circ \pi_{1, *}$ sur $(b)$ comme celle de $\sigma_{2} \otimes \sigma_{1} \in H_{d_{2}}\left(F_{2}\right) \otimes H_{d_{1}}\left(F_{1}\right)$. Une nouvelle application du Lemme 5.2.1 permet alors de conclure.

Nous prouvons maintenant par récurrence sur $m$ que $\varphi$ est identiquement nulle sur une base de dimension finie $m$, ce qui, compte tenu des remarques précédentes, se réduit à vérifier que $\varphi^{p}=0$ lorsque $d \leq p<d+m$. Dans le cas particulier où $m=0$, la question est déjà reglée. Supposons alors $\varphi$ nulle sur une base de dimension majorée par $m-1$ et soit $N$ de dimension $m$. Notons $N^{\prime}$ le $(m-1)$-squelette de $N$ et considérons la suite exacte longue d'homologie pour la paire $\left(N, N^{\prime}\right)$. Appliquons-lui $\varphi$. Grâce à la naturalité de l'intégration sur les fibres, on a le diagramme commutatif :

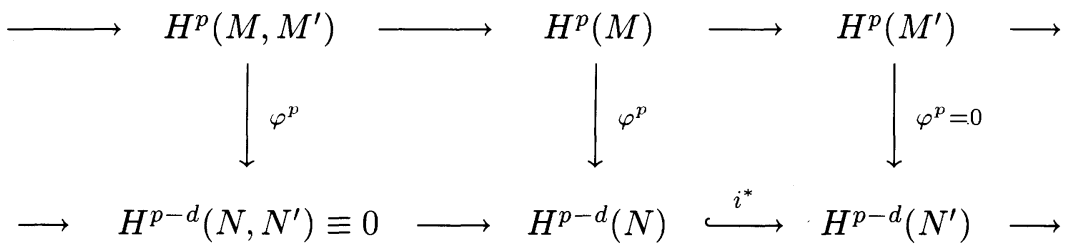

où $M^{\prime}=\pi^{-1}\left(N^{\prime}\right)$ et où $i^{*}$ est le morphisme associé à la restriction de $N$ à $N^{\prime}$. Ici, la flèche $\varphi^{p}$ de droite est nulle par hypothèse de récurrence et $H^{p-d}\left(N, N^{\prime}\right)$ est aussi nul puisqu'on suppose $p-d<m$. Ces remarques impliquent que $i^{*}$ est injective, ce qui entraîne la nullité de $\varphi^{p}: H^{p}(M) \rightarrow$ $H^{p-d}(N)$.

TOME $117-1989-\mathrm{N}^{\mathrm{O}} 2$ 
Le cas où $\mathrm{N}$ n'est pas de dimension finie résulte d'un argument évident par limite inductive.

5.3. Intégration sur les fibres en cohomologie équivariante. Soit $\pi: M \rightarrow N$ une fibration orientée de fibre $F$ de dimension $d$, soit $T$ un groupe compact, non nécessairement commutatif, et supposons $M$ et $N$ munis de structures de $T$-espaces rendant la fibration $T$-équivariante. Cette seule donnée supplémentaire va nous permettre de définir une opération d'intégration sur les fibres :

$$
\tilde{\pi}_{*}: H_{T}^{*}(M) \longrightarrow H_{T}^{*-d}(N),
$$

homomorphisme de $H_{T}^{*}(N)$-modules gradués, qui coïncide avec celle définie dans la section 5.1 lorsque $T=\{e\}$.

En effet, en posant $\tilde{\pi}([p, m])=[p, \pi(m)]$, on obtient une fibration $\tilde{\pi}: \mathbb{E} T \times^{T} M \rightarrow \mathbb{E} T \times \times^{T} N$ de fibre $F$ qui est orientable puisque l'on a le morphisme de fibrations de base $\mathbb{B} T$

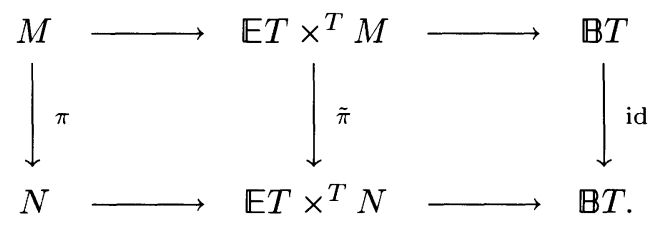

On sait, par la longue suite exacte d'homotopie, que le groupe fondamental $\Pi_{1}(N)$ se surjecte sur $\Pi_{1}\left(\mathbb{E} T \times{ }^{T} N\right)$. Cela implique que l'action de ce dernier est facteur de l'action (triviale par hypothèse) de $\Pi_{1}(N)$. La fibration $\tilde{\pi}$ est donc orientée et donne lieu à l'opération d'intégration sur les fibres en cohomologie équivariante en question.

Remarque. - En ajoutant aux données de la Proposition 5.2.1 des structures de $T$-espaces de façon à rendre les fibrations concernées $T$ équivariantes, la conclusion reste vraie en cohomologie équivariante.

5.4. Le cas différentiable. - Soit maintenant $M$ un CW-complexe orienté de dimension $d$ muni d'une structure de $T$-espace. La fibration équivariante $M \rightarrow\{\cdot\}$ donne lieu à un homomorphisme de $H_{T}^{*}(\cdot)$-modules gradués

$$
\int_{M}: H_{T}^{*}(M) \rightarrow H_{T}^{*-d}(\cdot)
$$

s'interprétant dans le contexte différentiel grâce aux éléments décrits dans les paragraphes suivants. 
5.4.1. - Supposons jusqu'à la fin de cette section le groupe $T$ connexe et d'algèbre de Lie $\mathfrak{t}$. Supposons aussi l'espace $M$ muni d'une structure de variété différentiable, compacte et orientée de dimension $d$, sur laquelle $T$ opère différentiablement à gauche. Notons $\left(\Omega^{*}(M), d\right)$ le complexe des formes différentielles sur $M$ à valeurs dans le corps des nombres complexes $\mathbb{C}$ et $S^{* / 2}\left(\mathfrak{t}^{2}\right)$ l'algèbre des applications polynômes sur $\mathfrak{t}$ à valeurs dans $\mathbb{C}$, graduée par la graduation double de la graduation habituelle. Considérons sur $S^{* / 2}\left(\mathfrak{t}^{\sim}\right)$ et sur $\Omega^{*}(M)$ les structures de $T$ algèbres naturelles et soit $S^{* / 2}\left(\mathfrak{t}^{\leftarrow}\right) \otimes \Omega^{*}(M)$ leur produit tensoriel. Notons $\left[S^{* / 2}\left(\mathfrak{t}^{\leftarrow}\right) \otimes \Omega^{*}(M)\right]^{T}$ la sous-algèbre bigraduée des éléments $T$-invariants. Celle-ci, munie de la graduation totale, notée $\Omega_{T}^{*}(M)$, est l'algèbre des formes différentielles $T$-équivariantes sur $M$. Ses éléments s'interprètent comme formes différentielles $\mu(Y)$ sur $M$ dépendant polynomialement d'un paramètre $Y \in \mathfrak{t}$ et vérifiant la condition d'équivariance $t \cdot \mu(Y)=$ $\mu(t \cdot Y)$, pour tous $t \in T$ et $Y \in \mathrm{t}$. Cette algèbre est munie d'une antidérivation $\delta$ de carré nul définie par

$$
(\delta \mu)(Y)=d(\mu(Y))+2 \pi \sqrt{-1} c(Y) \mu(Y)
$$

où $d$ et $c(Y)$ dénotent respectivement la différentielle extérieure et la contraction par le champ de vecteurs associé à l'action infinitésimale de $Y$ sur $M$ (cf. [9], [7]). Le couple $\left(\Omega_{T}^{*}(M), \delta\right)$ est donc un complexe et l'on doit à H. CARTAN ([9]) le fait que son homologie s'identifie canoniquement à la cohomologie $T$-équivariante de $M$ à coefficients complexes.

5.4.2. Suites spectrales. - La filtration $\left(\mathbb{B} T_{n}\right)_{n \geq 0}$ du classifiant de $T$ par les $n$-squelettes induit une filtration $\left(\mathbb{E} T_{n}\right)_{n \geq 0}$ du fibré universel en posant $\mathbb{E} T_{n}=p^{-1}\left(\mathbb{B} T_{n}\right)$. Il en résulte la filtration croissante $\left(\mathbb{E} T_{n} \times{ }^{T} M\right)_{n \geq 0}$ de l'espace $\mathbb{E} T \times^{T} M$, filtration qui donne lieu à la suite spectrale de Leray-Serre associée à la fibration $\mathbb{E} T \times{ }^{T} M \rightarrow \mathbb{B} T$

$$
E_{2}^{p, q} \equiv H^{p}\left(\mathbb{B} T ; H^{q}(M ; A)\right) \Longrightarrow H_{T}^{p+q}(M ; A),
$$

où $A$ dénote un module de coefficients. En particulier, si $A=\mathbb{C}$, nous avons

$$
E_{2}^{p, q} \equiv H^{p}(\mathbb{B} T ; \mathbb{C}) \otimes H^{q}(M ; \mathbb{C})
$$

Dans ce cas nous disposons aussi du complexe des formes différentielles $T$-équivariantes que nous allons filtrer par le degré polynôme en posant

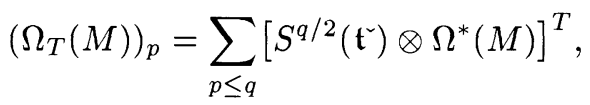

TOME $117-1989-\mathrm{N}^{\circ} 2$ 
ce qui fournit une suite spectrale du premier quadrant pour laquelle $d_{1}=0$ et dont le terme $E_{2}^{p, q}$ s'identifie trivialement à $S^{p / 2}\left(\mathfrak{t}^{\sim}\right)^{T} \otimes H^{q}(M ; \mathbb{C})$. On remarquera que d'après l'identification de $S^{* / 2}\left(\mathfrak{t}^{\complement}\right)^{T}$ à $H^{*}(M ; \mathbb{C})([8])$, on a un isomorphisme canonique entre les termes $E_{2}$ des deux suites spectrales considérées. Les techniques developées par H. CARTAN dans [9] permettent de préciser cette remarque en prouvant que cet isomorphisme est, en fait, le reflet d'un isomorphisme global des suites considérées dont l'intérêt est de fournir un lien entre les aspects purement algébriques et différentiels de nos considérations. La proposition suivante, qui intervient de manière essentielle dans les démonstrations de la section 3, en découle.

Proposition 5.4.2. - Soit $M$ une T-variété différentiable de dimension d, compacte, connexe et orientée. L'application $J_{M}$ de $\Omega_{T}^{*}(M) \grave{a}$ valeurs dans $\Omega_{T}^{*-d}(\cdot)=S^{(*-d) / 2}\left(\mathfrak{t}^{`}\right)^{T}$, définie par $J_{M}(\mu(Y))=\int_{M} \mu(Y)$ (ici le symbole $\int_{M}$ dénote l'intégration ordinaire) induit en cohomologie $T$-équivariante un homomorphisme de $S^{* / 2}\left(\mathfrak{t}^{\complement}\right)^{T}$-modules gradués qui coïncide avec l'opération d'integration sur les fibres associée à la fibration $T$-équivariante $M \rightarrow\{\cdot\}$ moyennant l'identification canonique de $H_{T}^{*}(\cdot ; \mathbb{C})$ et $S^{* / 2}\left(\mathfrak{t}^{\longleftarrow}\right)^{T}$.

Démonstration. - L'application $J_{M}$ est évidemment un homomorphisme de complexes filtrés (par le degré polynôme) de degré total $-d$ et induit donc un homomorphisme des suites spectrales associées qui est nul sur les termes $E_{2}^{p, q}$ lorsque $q<d$, et qui vaut $1 \otimes \int_{M}$ sur $S^{p / 2}\left(\mathfrak{t}^{\sim}\right)^{T} \otimes H^{d}(M)$. On reconnaît ainsi, moyennant l'identification des suites spectrales, la définition de l'intégration sur les fibres de la section 5.1.

5.5 Évaluation à l'origine. - Considérons à nouveau le terme $E_{2}^{p, q}$ de la suite spectrale associée à la fibration $\mathbb{E} T \times^{T} M \rightarrow \mathbb{B} T$. Les applications $d_{r}: E_{2}^{-r, q+r-1} \rightarrow E_{r}^{0, q}$, qui sont évidemment nulles, garantissent que $E_{\infty}^{0, q} \subseteq E_{2}^{0, q} \equiv H^{0}\left(\mathbb{B} T ; H^{q}(M)\right) \equiv H^{q}(M)$. On en déduit un homomorphisme d'algèbres $v_{0}(M): H_{T}^{*}(M) \rightarrow H^{*}(M)$, défini par la composition

$$
H_{T}^{*}(M) \longrightarrow H_{T}^{*}(M)_{0} / H_{T}^{*}(M)_{1} \longleftrightarrow H^{*}(M)
$$

que l'on appelle évaluation à l'origine. C'est par ailleurs l'homomorphisme induit en cohomologie par la fibration canonique $\nu(M): \mathbb{E} T \times M \rightarrow$ $\mathbb{E} T \times_{T} M$.

Corollaire 5.5.1. - Les opérations d'intègration sur les fibres et d'évaluation à l'origine commutent. 
Démonstration. - Soit $\pi: M \rightarrow N$ une fibration $T$-équivariante orientée de fibre $F$. Ce corollaire est une conséquence immédiate de la Proposition 5.1.1 appliquée au morphisme de fibrations $(\nu(M), \nu(N))$ de $($ id $\times \pi, \mathbb{E} T \times M, \mathbb{E} T \times N)$ dans $\left(\tilde{\pi}, \mathbb{E} T \times_{T} M, \mathbb{E} T \times_{T} N\right)$, qui donne l'égalité $v_{0}(N) \circ \tilde{\pi}_{*}=\pi_{*} \circ v_{0}(M)$.

Nous terminons cet appendice de rappels en justifiant, dans le lemme suivant, la terminologie d'évaluation à l'origine pour l'application $v_{0}$.

Lemme 5.5.1. - Soit $M$ une T-variété différentiable. Notons $\epsilon$ l'application de $\left(\Omega_{T}^{*}(M), \delta\right)$ à valeurs dans $\left(\Omega^{*}(M), d\right)$ définie par la condition $\epsilon(\mu(Y))=\mu(0)$. L'application $\epsilon$ passe en cohomologie où elle définit un homomorphisme d'algèbres qui coïncide avec $v_{0}(M)$.

Démonstration. - L'application $\epsilon$ est clairement un homomorphisme d'algèbres différentielles et passe donc bien en cohomologie. Munissons $\Omega_{T}^{*}(M)$ de sa filtration par degrés polynôme et $\Omega^{*}(M)$ de celle définie par $\Omega_{T}^{*}(M)_{0}=\Omega^{*}(M)$ et $\Omega_{T}^{*}(M)_{1}=0$. L'homomorphisme $\epsilon$ respecte ces filtrations et définit un morphisme sur les suites spectrales associées, $\tilde{E}$ et $E$ respectivement, où nous avons le diagramme commutatif suivant

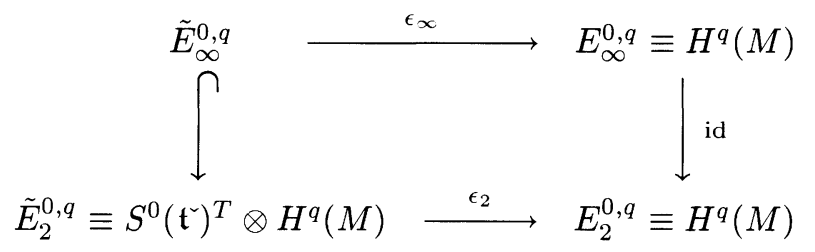

$\epsilon_{2}$ dénotant l'application évidente. On retrouve ainsi la définition de $v_{0}(M)$ donnée plus haut. 


\section{BIBLIOGRAPHIE}

[1] Arabia (A.). - Thèse de doctorat, Université de Paris VII, 1985.

[2] Arabia (A.). - Cycles de Schubert et cohomologie équivariante de $K / T$, Invent. Math., t. 85, 1986, p. 39-52.

[3] Arabia (A.). - Cohomologie $T$-équivariante de $G / B$ pour un groupe $G$ de Kač-Moody, C. R. Acad. Sc. Paris, t. 302, Série I, $\mathrm{n}^{\circ} 17,1986$.

[4] Arabia (A.). - Cohomologie T-équivariante de la variété de drapeaux d'un groupe de Kač-Moody, Préprint, École Polytechnique, 1986.

[5] Атіуан (M. F.) and Вотт (R). - The moment map and equivariant cohomology, Topology, t. 23, 1984, p. 1-28.

[6] Berline (N.) and Vergne (M.). - Fourier transforms of orbits of the coadjoint representation, Representation theory of reductive groups [Park City, Utah, 1982], pp. 53-67. - Prog. Math. 40, Birkhaüser, Boston, Mass. 1983.

[7] Berline (N.) et Vergne (M.). - Zéros d'un champ de vecteurs et classes caractéristiques équivariantes, Duke Math. J., t. 50, 1983 , p. 539-549.

[8] Borel (A.). - Sur la cohomologie des espaces fibrés principaux et des espaces homogènes de groupes de Lie compacts, Annals of Math., Vol. 57, $\mathrm{n}^{\circ} 1,1953$.

[9] Cartan (H.). - Notions d'algèbre différentielle, applications aux groupes de Lie... et La transgression dans un groupe de Lie et dans un espace fibré principal, Colloque de topologie algébrique, Bruxelles 1950, pp. 16-27 et pp. $57-71$.

[10] KAČ (V.). - Constructing groups associated to infinite dimensional Lie algebras, Infinite dimensional groups with applications, M. S. R. I. publications $\mathrm{n}^{\circ} 4$, Springer Verlag, 1985 .

[11] KAČ (V.) and Peterson (D.). - Cohomology of infinite dimensional groups and their flag varieties, à paraître.

[12] Kostant (B.) and Kumar (S.). - The Nil Hecke ring and cohomology of $G / P$ for a Kač-Moody group $G$, Proc. Nat. Acad. Sci. USA, t. 83, 1986, p. 1543.

[13] Kostant (B.) and Kumar (S.). - The Nil Hecke ring and cohomology of $G / P$ for a Kač-Moody group $G$, Advances in Math., t. 68, 1986, p. 187-237.

[14] Spanier (E. H.). - Algebraic topology. - 1966, Springer-Verlag, New York. 